Universidade de São Paulo

Instituto de Física

\title{
Quebra da Simetria de Lorentz e o Termo de Chern-Simons em $(3+1)$ Dimensões.
}

\author{
Liner de Souza Santos
}

Orientador: Prof. Dr. Adilson José da Silva

\section{COMISSÃO EXAMINADORA}

Prof.Dr. Adilson José da Silva (IFUSP)

Prof.Dr. Alex Gomes Dias (UFABC)

Prof.Dr. Fernando Tadeu Caldeira Brandt (IFUSP)

Dissertação de mestrado apresentada ao Instituto de Física para a obtenção do título de Mestre em Ciências.

São Paulo 2009 
Dedico aos meus amados pais,

Roberto e Ana Elizabete,

e ao valoroso Prof. Dr. Adilson José da Silva por sempre confiarem e me incentivarem. 


\section{AGRADECIMENTOS}

A Deus que é a dimensão mais poderosa da sabedoria, por conceder-me vida e condições para que eu conseguisse percorrer e cumprir esta importante etapa da minha vida..

Aos estimados companheiros do grupo de pesquisa, Professor Dr. Marcelo Gomes, Alysson, André, Bruno, Carlos, Denny, Fernando e Tiago.

Aos meus queridos irmãos Leandro, Leonardo, e Ana pelo apoio sempre dado.

Aos meus amigos do departamento, João Eduardo, Juliano, Leonardo, Roberto Parra e Walney.

À Amélia e Simone pela cordialidade e cooperação.

Ao Eber e à Francislene pelo bom atendimento sempre.

Aos meus amigos, sem os quais não teria chegado até aqui.

À CNPQ, pelo necessário apoio financeiro. 
"Um pouco de Ciência nos afasta de Deus. Muito, nos aproxima"(Louis Pasteur) 


\section{Sumário}

1 Introdução 1

2 Introdução à Relatividade Geral $\quad 5$

2.1 Princípio de Equivalência . . . . . . . . . . . . . 5

2.1.1 Força Gravitacional . . . . . . . . . . . . 6

2.1.2 Relação entre $g_{\mu \nu}$ e $\Gamma_{\mu \nu}^{\lambda} \ldots \ldots \ldots \ldots 10$

2.1.3 O Limite Newtoniano . . . . . . . . . . . . . 14

2.1 .4 Dilatação do tempo . . . . . . . . . . . . 16

2.1.5 Forma matricial da métrica . . . . . . . . . 19

2.2 Princípio de Covariância Geral . . . . . . . . . . . . 21

2.2.1 Vetores e Tensores . . . . . . . . . . . . 22

2.2 .2 Densidades Tensoriais . . . . . . . . . . 23

2.2.3 Transformação da Conexão Afim . . . . . . . . . 24

2.2.4 Derivada Covariante . . . . . . . . . . . 24

2.2 .5 Transporte paralelo .............. 26 
2.3 Tetradas .............................. 29

3 Férmions num Campo de Calibre 33

3.1 O Campo de Calibre de Chern-Simons . . . . . . . . . . 33

3.2 Propagador de Maxwell-Chern-Simons . . . . . . . . . . 35

3.3 Indução de Chern-Simons em $(2+1)$ D na Teoria de Calibre . . 39

3.3 .1 Informações úteis . . . . . . . . . . . . . . 39

4 Indução de Chern-Simons em $(3+1) \mathrm{D} \quad 47$

4.1 Teoria de Calibre . . . . . . . . . . . . . 47

4.1.1 Convenções Adotadas . . . . . . . . . . . . . . 47

4.1.2 Cálculo do Termo Induzido . . . . . . . . . . 48

4.2 Gravitação . . . . . . . . . . . . . . . . 56

5 Conclusão $\quad 63$

$\begin{array}{ll}\text { A Variáveis de Grassmann } & 65\end{array}$

B Prova da identidade $\ln \operatorname{det} B=\operatorname{tr} \ln B$

C Obtenção de $\operatorname{Tr} \mathcal{O}\left(\mathbf{x}, \partial_{X}\right)=\int \frac{d^{D} x}{(2 \pi)^{D}} \mathcal{O}\left(\partial_{x}+i k, x\right) \quad 73$

D Aproximação da série de Baker-Campbell-Hausdorff $\quad 77$

$\begin{array}{ll}\text { E Integrais D-dimensionais } & 81\end{array}$ 


\section{RESUMO}

Nesta dissertação estudamos o modelo de Chern-Simons na teoria de calibre e na gravitação. Para tanto, estudamos o problema de férmions interagindo num primeiro momento com um campo eletromagnético e num segundo momento, com um campo gravitacional. Introduzimos, em ambos os casos, um campo de fundo que quebra a simetria de Lorentz. Fizemos o cálculo da ação efetiva pelo método do tempo próprio de Schwinger, na aproximação de um laço e determinamos a ação efetiva, que contém os termos induzidos de Chern-Simons. 


\section{ABSTRACT}

In this dissertation, we studied the Chern-Simons model in the gauge and the gravitation theories. So, we study the problem of fermions interacting, in a first moment, with a electromagnetic field and in a second moment, with a gravitational field. We introduced in the interaction lagragian, for both cases, a background field which break the Lorentz simmetry.

We did the effective action calculus by the Schwinger proper time method, in the one loop approximation and we determined the effective action which the induced Chern-Simons terms. 


\section{Capítulo 1}

\section{Introdução}

Ao vermos a imagem projetada de um objeto tridimensional, observamos apenas seu contorno. Com certa tecnologia e informações prévias, podemos inferir sobre sua massa e provável constituição. Projetando em mais uma ou duas posições diferentes, conseguiremos descrever este objeto sem muitos detalhes, ou seja, conseguimos informações de um objeto tridimensional a partir de projeções num plano bidimensional.

Em teoria de campos, muitas vezes, ocorre algo semelhante, nos fornecendo informações importantes estudarmos um modelo formulado num espaço-tempo de dimensão $\mathrm{D}$ afim de obtermos uma teoria para um espaço-tempo de dimensão $\mathrm{D}+1$.

Um exemplo de uma teoria formulada em $(2+1)$ D é o modelo de ChernSimons [8], um modelo "topologicamente massivo" que em $(2+1)$ dimensões 
surge como uma complementação da eletrodinâmica de Maxwell. Tal complementação descreve fenômenos eletromagnéticos a curtas distâncias e o efeito Hall quântico.

Termos do tipo Chern-Simons puderam ser obtidos em teorias em $(3+1)$ dimensões, através da introdução de um campo de fundo na lagrangiana original, que quebra a simetria de Lorentz para que surjam os termos referentes ao modelo de Chern-Simons. Um dos pontos importantes desse modelo é a existencia de campos de calibre massivos (o fóton passa a ter uma massa diferente de zero) ${ }^{1}$. Embora ainda não tenha sido vista experimentalmente uma quebra da invariância de Lorentz, teorias envolvendo tal fato nos últimos anos têm sido sugeridas e estudadas $[9,7]$.

Além disso, com o ressurgimento da suposição de que o espaço-tempo possa ser não comutativo, o que implicaria numa quebra da simetria de Lorentz, tem crescido o número de publicações que contêm essa violação.

Nesta dissertação, buscamos uma possível indução de termos do tipo ChernSimons no caso de um campo fermiônico acoplado a um campo de calibre e a um campo gravitacional para um espaço-tempo quadridimensional, através da introdução na lagrangiana de interação, de um campo de fundo constante que quebra a simetria de Lorentz. Calculamos a ação efetiva de ambas interações, fazendo a regularização das integrais a partir do método do tempo

\footnotetext{
${ }^{1} \mathrm{~A}$ compatibilidade com os resultados experimentais atuais para a massa do fóton impõem o limite $m<10^{-18} \mathrm{eV}$
} 
próprio [12], como feito anteriormente em $(2+1)$ dimensões [16], verificando a possível indução de termos do tipo Chern-Simons.

No próximo capítulo, é apresentada uma revisão da relatividade geral, com os principais tópicos que serão utilizados no decorrer da dissertação. No terceiro capítulo, a teoria de Chern-Simons é introduzida como uma teoria de calibre em $(2+1)$ D e são obtidos os termos induzidos na ação efetiva. No capítulo 4 são calculadas as ações efetivas da teoria de calibre e da Gravitação com a presença de um campo de fundo que quebra a simetria de Lorentz. Finalmente no capítulo 5 são feitas a conclusão e a análise do trabalho. 


\section{Capítulo 2}

\section{Introdução à Relatividade Geral}

\subsection{Princípio de Equivalência}

Este é o princípio básico da teoria da Relatividade Geral e em poucas palavras diz que dado um ponto qualquer numa região do espaço com um campo gravitacional arbitrário, é possível escolher um referencial localmente inercial, tal que numa região suficientemente pequena em torno deste ponto, as leis da natureza têm a mesma forma que em um referencial cartesiano desacelerado numa região sem campo gravitacional. Podemos fazer uma analogia deste princípio com um axioma devido a Gauss, no qual ele diz que dado um ponto numa superfície curva, podemos construir um referencial cartesiano(plano) tal que nas proximidades deste ponto, valem os princípios da geometria euclidiana. 


\subsubsection{Força Gravitacional}

Consideremos uma partícula movendo-se livremente somente sob influência do campo gravitacional. De acordo com o princípio de equivalência, há um sistema de coordenadas $\xi^{\alpha}$ caindo livremente (isto é, $\xi^{\alpha}$ é o sistema localmente inercial), no qual a equação de movimento da partícula é:

$$
\frac{d^{2} \xi^{\alpha}}{d \tau^{2}}=0
$$

sendo $d \tau$ o intervalo entre dois eventos ocorrendo com a partícula(intervalo de tempo próprio da partícula), definido por

$$
d \tau^{2}=-\eta_{\alpha \beta} d \xi^{\alpha} d \xi^{\beta}
$$

onde $\eta_{\alpha \beta}$ é o tensor métrico de Minkowski. Fixemos agora um referencial arbitrário no laboratório, que chamaremos de $x^{\alpha}$ (ou seja, $x^{\alpha}$ é não inercial). Podemos escrever o sistema de coordenadas em queda livre como função deste e vice-versa. Aplicando isso na Eq.(2.1):

$$
\begin{aligned}
& \frac{d}{d \tau}\left(\frac{\partial \xi^{\alpha}}{\partial x^{\mu}} \frac{d x^{\mu}}{d \tau}\right)=0 \\
& \frac{\partial \xi^{\alpha}}{\partial x^{\mu}} \frac{d^{2} x^{\mu}}{d \tau^{2}}+\frac{\partial^{2} \xi^{\alpha}}{\partial x^{\mu} \partial \tau} \frac{d x^{\mu}}{d \tau}=\frac{\partial \xi^{\alpha}}{\partial x^{\mu}} \frac{d^{2} x^{\mu}}{d \tau^{2}}+\frac{\partial^{2} \xi^{\alpha}}{\partial x^{\mu} \partial x^{\nu}} \frac{d x^{\nu}}{d \tau} \frac{d x^{\mu}}{d \tau}=0 \\
& \frac{d^{2} x^{\lambda}}{d \tau^{2}}+\Gamma_{\mu \nu}^{\lambda} \frac{d x^{\mu}}{d \tau} \frac{d x^{\nu}}{d \tau}=0
\end{aligned}
$$

Esta última equação descreve o movimento de uma partícula em queda livre num referencial arbitrário $x^{\alpha}$. A grandeza $\Gamma_{\mu \nu}^{\lambda}$ é chamada de conexão afim e pode ser entendida como uma "correção"às equações devido à curvatura do 
espaço-tempo:

$$
\Gamma_{\mu \nu}^{\lambda} \equiv \frac{\partial^{2} \xi^{\alpha}}{\partial x^{\mu} \partial x^{\nu}} \frac{\partial x^{\lambda}}{\partial \xi^{\alpha}}
$$

O tempo próprio também pode ser expresso em termos do sistema de coordenadas $x^{\mu}$, como segue:

$$
d \tau^{2}=-\eta_{\alpha \beta} \frac{\partial \xi^{\alpha}}{\partial x^{\mu}} d x^{\mu} \frac{\partial \xi^{\beta}}{\partial x^{\nu}} d x^{\nu} \equiv-g_{\mu \nu} d x^{\mu} d x^{\nu}
$$

onde definimos o chamado tensor métrico $g_{\mu \nu}$ :

$$
g_{\mu \nu} \equiv \eta_{\alpha \beta} \frac{\partial \xi^{\alpha}}{\partial x^{\mu}} \frac{\partial \xi^{\beta}}{\partial x^{\nu}}
$$

Para uma partícula sem massa, a equação de movimento é análoga à (2.1), exceto pelo fato que a variável independente não pode ser o tempo próprio como na Eq. (2.5) pois se a massa da partícula é nula, o lado direito de (2.5) anula-se. Então, no lugar de $\tau$, podemos usar $\sigma \equiv \xi^{0}$, tal que:

$$
\begin{aligned}
& \frac{d^{2} \xi^{\alpha}}{d \sigma^{2}}=0 \\
& 0=-\eta_{\alpha \beta} \frac{d \xi^{\alpha}}{d \sigma} \frac{d \xi^{\beta}}{d \sigma}
\end{aligned}
$$

E a partir desses resultados, chegamos à equação de movimento para uma partícula sem massa (por exemplo, um fóton) num campo gravitacional e num sistema de coordenadas arbitrário:

$$
\begin{aligned}
& \frac{d^{2} x^{\mu}}{d \sigma^{2}}+\Gamma_{\nu \lambda}^{\mu} \frac{d x^{\nu}}{d \sigma} \frac{d x^{\lambda}}{d \sigma}=0 \\
& -g_{\mu \nu} \frac{d x^{\mu}}{d \sigma} \frac{d x^{\nu}}{d \sigma}=0 .
\end{aligned}
$$


Afortunadamente, não precisamos conhecer $\tau$ e $\sigma$ para determinar a dinâmica da partícula, seja ela massiva ou não, pois as equações de movimento para ambos os casos nos dão $x^{\mu}(\tau)$ ou $x^{\mu}(\sigma)$ e esses parâmetros podem ser eliminados e $x^{\mu}$ escrito como $x^{\mu}(t)$. Chamemos a atenção para as equações (2.6) e (2.10) que aparentemente são muito semelhantes mas com propósitos distintos: a Eq.(2.6) nos diz como calcular o tempo próprio, enquanto a Eq.(2.10) nos diz que o tempo $d t$ gasto por um fóton para percorrer uma distância $d \vec{x}$ é determinado pela equação quadrática em $d x^{0}$, lembrando que $x^{0}=t:$

$$
\begin{aligned}
& 0=-g_{\mu \nu} \frac{d x^{\mu}}{d \sigma} \frac{d x^{\nu}}{d \sigma} \Rightarrow g_{00}\left(d x^{0}\right)^{2}+2 g_{i 0} d x^{i} d x^{0}+g_{i j} d x^{i} d x^{j}=0 \\
& d t=\frac{1}{g_{00}}\left[-g_{i 0} d x^{i}-\left\{\left(g_{i 0} g_{j 0}-g_{i j} g_{00}\right) d x^{i} d x^{j}\right\}^{1 / 2}\right] .
\end{aligned}
$$

Para saber o tempo gasto pela partícula sem massa percorrer uma certa distância ao longo de qualquer trajetória, basta integrar a expressão acima ao longo da trajetória. Agora que temos em mãos as expressões para o tensor métrico e para a conexão afim, podemos calculá-los para um ponto qualquer X no sistema de coordenadas arbitrário $x^{\mu}$ e a partir daí determinar as coordenadas localmente inerciais $\xi^{\alpha}(x)$ numa vizinhança de X. Primeiramente, multiplicamos (2.4) por $\frac{\partial \xi^{\beta}}{\partial x^{\lambda}}$ e usamos que:

$$
\begin{aligned}
& \frac{\partial \xi^{\beta}}{\partial x^{\lambda}} \frac{\partial x^{\lambda}}{\partial \xi^{\alpha}}=\delta_{\alpha}^{\beta} . \\
& \frac{\partial \xi^{\beta}}{\partial x^{\lambda}} \Gamma_{\mu \nu}^{\lambda}=\delta_{\alpha}^{\beta} \frac{\partial^{2} \xi^{\alpha}}{\partial x^{\mu} \partial x^{\nu}}=\frac{\partial^{2} \xi^{\beta}}{\partial x^{\mu} \partial x^{\nu}} .
\end{aligned}
$$


Chegamos a uma equação diferencial para $\xi^{\alpha}$, que resolveremos a seguir: Como estamos interessados na vizinhança de $\mathrm{X}$, supomos que $x^{\mu}=X^{\mu}+\epsilon^{m u}$

$$
\begin{aligned}
& \xi^{\alpha}(x)=\xi^{\alpha}(X+\epsilon) \approx \xi^{\alpha}(X)+\epsilon^{\mu} \frac{\partial \xi^{\alpha}(X)}{\partial X^{\mu}}+ \\
& \epsilon^{\mu} \epsilon^{\nu} 2 \frac{\partial^{2} \xi^{\alpha}(X)}{\partial X^{\mu} \partial X^{\nu}}+\frac{1}{3 !} \epsilon^{\mu} \epsilon^{\nu} \epsilon^{\rho} \frac{\partial^{3} \xi^{\alpha}(X)}{\partial X^{\mu} \partial X^{\nu} \partial X^{\rho}}+\ldots
\end{aligned}
$$

E podemos verificar que teremos apenas dois coefcientes independentes, a saber,

$$
\begin{aligned}
& a^{\alpha} \equiv \xi^{\alpha}(X) \\
& b_{\mu}^{\alpha} \equiv \frac{\partial \xi^{\alpha}(X)}{\partial X^{\mu}} .
\end{aligned}
$$

E também descobrimos o comportamento do tensor métrico nas vizinhanças de X:

$$
g_{\mu \nu}(X)=\eta_{\alpha \beta} b_{\mu}^{\alpha} b_{\nu}^{\beta}
$$

que pode ser verificado por (2.6).

Assim, dados $\Gamma_{\mu \nu}^{\lambda}$ e $g_{\mu \nu}$ em X, o sistema de coordenadas localmente inercial $\xi^{\alpha}$ é parcialmente determinado em ordem de $(x-X)^{2}$, exceto por uma ambigüidade entre as constantes $a^{\alpha}$ e $b_{\lambda}^{\alpha}$. A constante $b^{\alpha}$ é determinada por (2.15) mesmo sob uma transformação de Lorentz: $b_{\lambda}^{\alpha} \rightarrow \Lambda_{\beta}^{\alpha} b_{\lambda}^{\beta}$. Então, a ambigüidade entre as constantes apenas reflete o fato que se $\xi^{\alpha}$ são coordenadas localmente inerciais, as coordenadas $\xi^{\prime \alpha}=\Lambda_{\bullet \beta}^{\alpha} \xi^{\beta}+c^{\alpha}$ também serão e conseqüentemente, desde que $\Gamma_{\mu \nu}^{\lambda}$ e $g_{\mu \nu}$ determinam o sistema de coordenadas 
localmente inercial, mesmo sob uma transformação de Lorentz não homogênea, e lembrando que o campo gravitacional não exerce nenhum efeito sobre esse sistema, poderíamos pensar que todos os efeitos devidos à gravidade estão concentrados nesses dois tensores. No entanto, a eq.(2.13) determina as coordenadas somente para $\mathrm{x}=\mathrm{X}$ e para pontos na vizinhança. Para determinarmos as coordenadas para qualquer x, são necessárias aproximações mais complexas na série perturbativa, envolvendo derivadas da conexão afim.

\subsubsection{Relação entre $g_{\mu \nu}$ e $\Gamma_{\mu \nu}^{\lambda}$}

Nosso tratamento da partícula em queda livre tem nos mostrado que a grandeza (campo) que determina a força gravitacional é a conexão afim, ao passo que o tempo próprio é determinado pelo tensor métrico. Iremos mostrar agora que o tensor métrico é o potencial gravitacional, isto é, as derivadas do tensor métrico determinam o campo $\Gamma_{\mu \nu}^{\lambda}$ Primeiramente, derivemos a expressão do tensor métrico com respeito a $x^{\lambda}$ (Eq.(2.6)):

$$
\begin{aligned}
& \frac{\partial g_{\mu \nu}}{\partial x^{\lambda}}=\frac{\partial^{2} \xi^{\alpha}}{\partial x^{\mu} \partial x^{\lambda}} \frac{\partial \xi^{\beta}}{\partial x^{\nu}} \eta_{\alpha \beta}+\frac{\partial \xi^{\alpha}}{\partial x^{\mu}} \frac{\partial^{2} \xi^{\beta}}{\partial x^{\lambda} \partial x^{\nu}} \eta_{\alpha \beta} \\
& =\left[\Gamma_{\lambda \mu}^{\rho} \frac{\partial \xi^{\beta}}{\partial x^{\nu}} \frac{\partial \xi^{\alpha}}{\partial x^{\rho}}+\Gamma_{\lambda \nu}^{\rho} \frac{\partial \xi^{\alpha}}{\partial x^{\mu}} \frac{\partial \xi^{\beta}}{\partial x^{\rho}}\right] \eta_{\alpha \beta} \\
& \frac{\partial g_{\mu \nu}}{\partial x^{\lambda}}=\Gamma_{\lambda \mu}^{\rho} g_{\rho \nu}+\Gamma_{\lambda \nu}^{\rho} g_{\rho \mu} .
\end{aligned}
$$


Somemos em ambos os lados a mesma derivada do tensor métrico com $\mu \leftrightarrow \lambda$ e subtraiamos a mesma derivada $\operatorname{com} \nu \leftrightarrow \lambda$, com isso, temos:

$$
\begin{aligned}
& \frac{\partial g_{\mu \nu}}{\partial x^{\lambda}}+\frac{\partial g_{\lambda \nu}}{\partial x^{\mu}}-\frac{\partial g_{\mu \lambda}}{\partial x^{\nu}}= \\
& =\Gamma_{\lambda \mu}^{\rho} g_{\rho \nu}+\Gamma_{\lambda \nu}^{\rho} g_{\rho \mu}+\Gamma_{\mu \lambda}^{\alpha} g_{\alpha \nu}+\Gamma_{\mu \nu}^{\alpha} g_{\alpha \lambda}-\Gamma_{\nu \mu}^{\alpha} g_{\alpha \lambda}-\Gamma_{\nu \lambda}^{\alpha} g_{\alpha \mu}= \\
& =2 \Gamma_{\lambda \mu}^{\rho} g_{\rho \nu} .
\end{aligned}
$$

Definamos agora uma matriz $g^{\nu \sigma}$ como a inversa de $g_{\nu \sigma}$,ou seja, $g^{\nu \sigma}$ é tal que $g_{\nu \sigma} g^{\nu \rho}=\delta_{\sigma}^{\rho}$. Assim, Contraindo (2.18) com a identidade acima, obtemos:

$$
\begin{aligned}
& g^{\nu \sigma}\left(\frac{\partial g_{\mu \nu}}{\partial x^{\lambda}}+\frac{\partial g_{\lambda \nu}}{\partial x^{\mu}}-\frac{\partial g_{\mu \lambda}}{\partial x^{\nu}}\right)=2 \Gamma_{\lambda \mu}^{\rho} g_{\rho \nu} g^{\nu \sigma} \\
& \Gamma_{\lambda \mu}^{\sigma}=\frac{1}{2} g^{\nu \sigma}\left(\frac{\partial g_{\mu \nu}}{\partial x^{\lambda}}+\frac{\partial g_{\lambda \nu}}{\partial x^{\mu}}-\frac{\partial g_{\mu \lambda}}{\partial x^{\nu}}\right)
\end{aligned}
$$

que é chamado Símbolo de Christoffel.

Uma conseqüência importante da relação entre a conexão afim e o tensor métrico é que a equação de movimento da partícula em queda livre mantém a forma do tempo próprio $d \tau$. Da expressão (2.5), vemos que:

$$
1=g_{\mu \nu} \frac{d x^{\mu}}{d \tau} \frac{d x^{\nu}}{d \tau}
$$

Ou seja,

$$
\begin{aligned}
& 0=\frac{d}{d \tau}\left[g_{\mu \nu} \frac{d x^{\mu}}{d \tau} \frac{d x^{\nu}}{d \tau}\right]= \\
& \frac{\partial g_{\mu \nu}}{\partial x^{\lambda}} \frac{d x^{\lambda}}{d \tau} \frac{d x^{\mu}}{d \tau} \frac{d x^{\nu}}{d \tau}+g_{\mu \nu} \frac{d^{2} x^{\mu}}{d \tau^{2}} \frac{d x^{\nu}}{d \tau}+g_{\mu \nu} \frac{d x^{\mu}}{d \tau} \frac{d^{2} x^{\nu}}{d \tau^{2}}= \\
& =\frac{\partial g_{\mu \nu}}{\partial x^{\lambda}} \frac{d x^{\lambda}}{d \tau} \frac{d x^{\mu}}{d \tau} \frac{d x^{\nu}}{d \tau}-g_{\mu \nu} \Gamma_{\alpha \beta}^{\mu} \frac{d x^{\alpha}}{d \tau} \frac{d x^{\beta}}{d \tau} \frac{d x^{\nu}}{d \tau}-g_{\mu \nu} \frac{d x^{\mu}}{d \tau} \Gamma_{\alpha \beta}^{\nu} \frac{d x^{\alpha}}{d \tau} \frac{d x^{\beta}}{d \tau}= \\
& =\left[\frac{\partial g_{\alpha \beta}}{\partial x^{\lambda}}-g_{\mu \lambda} \Gamma_{\alpha \beta}^{\mu}-g_{\lambda \nu} \Gamma_{\alpha \beta}^{\nu}\right] \frac{d x^{\alpha}}{d \tau} \frac{d x^{\beta}}{d \tau} \frac{d x^{\lambda}}{d \tau} .
\end{aligned}
$$


O termo entre colchetes é igual a:

$$
\frac{\partial g_{\alpha \beta}}{\partial x^{\lambda}}-2 g_{\mu \lambda} \Gamma_{\alpha \beta}^{\mu}=\frac{\partial g_{\alpha \beta}}{\partial x^{\lambda}}-\frac{\partial g_{\beta \lambda}}{\partial x^{\alpha}}-\frac{\partial g_{\alpha \lambda}}{\partial x^{\beta}}+\frac{\partial g_{\beta \alpha}}{\partial x^{\lambda}}
$$

Devido à simetria do tensor métrico e das derivadas de x, a eq.(2.21) se anula, ou seja,

$$
g_{\mu \nu} \frac{d x^{\mu}}{d \tau} \frac{d x^{\nu}}{d \tau}=-C
$$

onde $\mathrm{C}$ é uma constante do movimento. Por isso, uma vez escolhidas condições iniciais tais que $d \tau^{2}$ seja dado pela eq.(2.5), temos que $\mathrm{C}=1$ e que ela continua determinando a trajetória da partícula massiva. No caso de uma partćula sem massa, as condições iniciais dizem que $\mathrm{C}=0$ e a equação de movimento será zero ao longo da trajetória, uma vez que o tempo próprio é nulo para essas partćulas.

Como ilustração ${ }^{1}$, formularemos o movimento da partícula em queda livre como um princípio variacional, verificando que o pincípio de energia mínima continua válido. Assim, seja $\Delta t$ o tempo próprio "gasto"pela partícula para "cair"de um ponto A a um ponto B.

$$
\Delta t=\int_{A}^{B} \frac{d \tau}{d p} d p=\int_{A}^{B}\left[-g_{\mu \nu} \frac{d x^{\mu}}{d p} \frac{d x^{\nu}}{d p}\right]^{1 / 2} d p
$$

Façamos uma variação na trajetória de $x^{\mu}(p)$ para $x^{\mu}(p)+\delta x^{\mu}(p)$, fixando

\footnotetext{
${ }^{1}$ não é necessária a leitura do fim desta seção para entendimento do trabalho.
} 
condições de contorno como $\delta x^{\mu}\left(p_{A}\right)=\delta x^{\mu}\left(p_{b}\right)=0$

$$
\begin{aligned}
& \delta(\Delta t)=\int_{A}^{B} \delta f^{1 / 2} d p=\int_{A}^{B} d f^{1 / 2} d p \\
& d f^{1 / 2}=\frac{d f^{1 / 2}}{d f} d f=\frac{1}{2} f^{-1 / 2} d f \\
& \delta(\Delta t)=\frac{1}{2} \int_{A}^{B}\left[-g_{\mu \nu} \frac{d x^{\mu}}{d p} \frac{d x^{\nu}}{d p}\right]^{-1 / 2}\left[-\frac{\partial g_{\mu \nu}}{\partial x^{\lambda}} \delta x^{\lambda} \frac{d x^{\mu} d x^{\nu}}{d p d p}-2 g_{\mu \nu} \frac{d \delta x^{\mu} d x^{\nu}}{d p d p}\right] d p= \\
& =-\int_{A}^{B}\left[\frac{1}{2} \frac{\partial g_{\mu \nu}}{\partial x^{\lambda}} \delta x^{\lambda} \frac{d x^{\mu} d x^{\nu}}{d \tau d \tau}+g_{\mu \nu} \frac{d \delta x^{\mu} d x^{\nu}}{d \tau d \tau}\right] d \tau .
\end{aligned}
$$

Calculemos o segundo termo da integral acima:

$$
\begin{aligned}
& \frac{d \delta x^{\mu} d x^{\nu}}{d \tau d \tau}=\frac{d}{d \tau}\left(\delta x^{\mu} \frac{d x^{\nu}}{d \tau}\right)-\delta x^{\mu} \frac{d^{2} x^{\nu}}{d \tau^{2}} \\
& \int_{A}^{B} g_{\mu \nu} \frac{d \delta x^{\mu} d x^{\nu}}{d \tau d \tau}, \int_{A}^{B}\left[g_{\mu \nu} \frac{d}{d \tau}\left(\delta x^{\mu} \frac{d x^{\nu}}{d \tau}+\delta x^{\mu} \frac{d^{2} x^{\nu}}{d \tau^{2}}\right)\right] d \tau= \\
& =-\int_{A}^{B} \frac{\partial g_{\lambda \nu}}{\partial \tau} \delta x^{\lambda} \frac{d x^{\nu}}{d \tau} d \tau-\int_{A}^{B} \delta x^{\lambda} g_{\lambda \nu} \frac{d^{2} x^{\nu}}{d \tau^{2}} d \tau
\end{aligned}
$$

ressaltando que na última passagem foi feita uma integração por partes e foram usadas as condições de contorno. Substituindo (2.25) em (2.24) temos:

$$
\delta(\Delta t)=-\int_{A}^{B}\left[\frac{1}{2} \frac{\partial g_{\mu \nu}}{\partial x^{\lambda}} \frac{d x^{\mu} d x^{\nu}}{d \tau d \tau}-\frac{\partial g_{\lambda \nu}}{\partial x^{\sigma}} \frac{d x^{\sigma} d x^{\nu}}{d \tau d \tau}-g_{\lambda \nu} \frac{d^{2} x^{\nu}}{d \tau^{2}}\right] d \tau
$$

e lembrando a definição da conexão afim,

$$
\begin{aligned}
& \frac{1}{2} \frac{\partial g_{\mu \sigma}}{\partial x^{\lambda}}-\frac{\partial g_{\lambda \mu}}{\partial x^{\sigma}}=\frac{1}{2} \frac{\partial g_{\mu \sigma}}{\partial x^{\lambda}}-\frac{1}{2} \frac{\partial g_{\lambda \mu}}{\partial x^{\sigma}}-\frac{\partial g_{\sigma \lambda}}{\partial x^{\mu}}= \\
& =-g_{\rho \lambda} \Gamma_{\mu \sigma}^{\rho}
\end{aligned}
$$

Substituindo (2.27) em (2.26) obtemos:

$$
\delta(\Delta t)=\int_{A}^{B}\left[\frac{d^{2} x^{\nu}}{d \tau^{2}}+\Gamma_{\mu \sigma}^{\nu} \frac{d x^{\mu} d x^{\sigma}}{d \tau d \tau}\right] g_{\lambda \nu} \delta x^{\lambda} d \tau
$$


em que o integrando é a equação de movimento da partícula, sendo portanto nulo. $\delta(\Delta t)=0$

Ou seja, uma partícula em queda livre num espaço-tempo curvo mover-se-á de um ponto a outro pelo caminho de menor ou maior comprimento possível, representado pela equação $\left(\frac{d^{2} x^{\mu}}{d \tau^{2}}+\Gamma \ldots\right)=0$. Esses caminhos extremantes são denominados geodésicas e são trajetórias no espaço-tempo (ou seja trata-se do tempo próprio).

\subsubsection{O Limite Newtoniano}

2 Em algumas situações, podemos verficar que a geometria do espaçotempo sofre apenas uma deformação leve com relação à geometria euclidiana. Para verificarmos isso, consideremos uma partícula movendo-se vagarosamente (i.e. $v \ll c$ ), numa região com um campo gravitacional estacionário fraco, nesse caso, podemos considerar $\frac{d x^{i}}{d \tau} \ll \frac{d x^{0}}{d \tau}$ e aproximar a equação de movimento como:

$$
\frac{d^{2} x^{\mu}}{d \tau^{2}}+\Gamma_{00}^{\mu} \frac{d x^{0} d x^{0}}{d \tau d \tau}=0
$$

e como consideramos o campo gravitacional como estacionário, as derivadas temporais do tensor métrico anular-se-ão e:

$$
\Gamma_{00}^{\mu}=-\frac{1}{2} g^{\mu \nu} \frac{\partial g_{00}}{\partial x^{\nu}}
$$

\footnotetext{
${ }^{2}$ A leitura desta seção não é necessária para etendimento do trabalho, podendo ser suprimida.
} 
Como estamos também considerando o campo fraco, podemos adotar o sistema de coordenadas cartesiano, com uma pequena perturbação, isto é,

$$
g_{\alpha \beta}=\eta_{\alpha \beta}+h_{\alpha \beta}
$$

com: $\left|h_{\alpha \beta}\right| \ll\left|\eta_{\alpha \beta}\right|$. Disto segue que:

$$
\begin{aligned}
& \Gamma_{00}^{\alpha}=-\frac{1}{2} \eta^{\alpha \beta} \frac{\partial h_{00}}{\partial x^{\beta}}+\mathcal{O}\left(h^{2}\right) \\
& \frac{d^{2} x^{\alpha}}{d \tau^{2}} \approx \frac{1}{2} \eta^{\alpha \beta} \frac{\partial h_{00}}{\partial x^{\beta}} .
\end{aligned}
$$

Separarando agora na equação de movimento a parte espacial da parte temporal:

$$
\begin{aligned}
& \frac{d^{2} x^{0}}{d \tau^{2}}=\frac{1}{2} \eta^{0 \beta} \frac{\partial h_{00}}{\partial x^{\beta}}\left(\frac{d x^{0}}{d \tau}\right)^{2}=\frac{1}{2} \partial_{0} h_{00}\left(\frac{d x^{0}}{d \tau}\right)^{2}=0 . \\
& -\frac{d^{2} \vec{x}}{d \tau^{2}}=\frac{1}{2} \eta^{i \beta} \partial_{\beta} h_{00}\left(\frac{d x^{0}}{d \tau}\right)^{2}=\frac{1}{2} \nabla h_{00}\left(\frac{d x^{0}}{d \tau}\right)^{2} \\
& \frac{d^{2} \vec{x}}{d t^{2}}=\frac{1}{2} \nabla h_{00} .
\end{aligned}
$$

Da Mecânica Newtoniana, nós sabemos que

$$
\frac{d^{2} x}{d t^{2}}=-\nabla \phi
$$

onde $\phi$ é o potencial gravitacional, que a uma distância $r$ do centro de um corpo com simetria esférica e massa $\mathrm{M}$, tem a forma

$$
\phi=-\frac{G M}{r} .
$$


Comparando-se (2.33) e (2.32), temos ${ }^{3}$ :

$$
h_{00}=-2 \phi+C t e .
$$

E, sabemos que a grandes distâncias, o sistema deve obedecer à métrica de Minkowski, ou seja,

$$
\lim _{r \rightarrow \infty} h_{00}=0
$$

e como o potencial também deve anular-se no infinito, a constante deve ser zero e $h_{00}=-2 \phi$, ou seja

$$
g_{00}=-(1+2 \phi)
$$

O potencial gravitacional na superfície da Terra é da ordem de $10^{-9}$, na superfície de uma anã branca, que é um dos objetos mais massivos dos quais podemos tirar dados observacionais, é da ordem de $10^{-4}[10]$, ou seja, a distorção provocada na métrica devido à gravidade é em geral muito pequena.

\subsubsection{Dilatação do tempo}

${ }^{4}$ Considere dois relógios R1 e R2 iguais, sincronizados e parados no campo gravitacional, cujas coordenadas são, respectivamente x e y com relação ao

\footnotetext{
${ }^{3}$ Estamos nos referindo ao potencial adimensional que é a razão entre a energia potencial gravitacional $\frac{G M m}{r}$ e a energia de repouso relativística $m c^{2}$

${ }^{4} \mathrm{~A}$ leitura desta seção não é necessária para etendimento do trabalho, podendo ser suprimida.
} 
centro do campo. Esses relógios, emitem a cada instante fixo de tempo um sinal luminoso e cada um tem um observador O1 e O2 que estão respectivamente com R1 e R2. O tempo próprio do relógio R1 é dado por:

$$
\begin{aligned}
& d \tau=\left(-g_{\alpha \beta} d r^{\alpha} d r^{\beta}\right)^{1 / 2} \\
& =\left(-g_{00}\left(d r^{0}\right)^{2}-2 g_{i 0} d r^{0} d r^{i}-g_{i j} d r^{i} d r^{j}\right)^{1 / 2} \\
& =\left(-g_{00}\right)^{1 / 2} d r^{0},
\end{aligned}
$$

onde o quadrivetor $\mathrm{r}$ é um quadrivetor espaço temporal genérico e lembrando que os últimos dois termos na segunda linha são nulos pois os relógios estão parados. De acordo com a expressão acima, O1 medirá o tempo $d x^{0}$ entre dois sinais emitidos pelo relógio R1 dado por:

$$
d x^{0}=\frac{d \tau}{\left(g_{00}(x)\right)^{1 / 2}},
$$

e analogamente, o tempo medido por $\mathrm{O} 2$ entre dois sinais consecutivos emitidos por R2 é dado por:

$$
d y^{0}=\frac{d \tau}{\left(g_{00}(y)\right)^{1 / 2}}
$$

Como o campo gravitacional é estático, O2 medirá o mesmo intervalo de tempo entre dois sinais emitidos por R1 que o medido por O2. Ou seja, apenas um relógio é insuficiente para medir alguma alteraç ao no tempo devido à gravidade. Mas, se O2, observar o tempo entre dois sinais emitidos 
por R2 e depois por R1 e comparar ambos, obterá o seguinte resultado:

$$
\frac{d y_{R 1 / O 2}^{0}}{d y_{R 2 / O 2}^{0}}=\sqrt{\frac{g_{00}(y)}{g_{00}(x)}},
$$

o que indica uma alteração no tempo devido à gravidade. Se aplicarmos as equações acima para calcular o desvio de freqüência observado na Terra de um raio de luz emitido no sol devido a uma transição atômica, verificaremos que a luz sofre um desvio para o vermelho de aproximadamente $2.10^{-6}$, ou seja, duas partes por milhão quando comparada com a luz emitida pela mesma transição na Terra. Com isso nós vemos que é muito difícil detectar esse efeito devido ao potencial gravitacional do Sol, pois esse desvio é muito pequeno e pode ser mascarado por outros efeitos como convecção de gases na atmosfera do Sol, temperatura e também devido à rotação do Sol. Fatores que, além de tornar o efeito gravitacional não observável, podem provocar desvios para o azul.

Assim, para verificarmos esse efeito, devemos procurar corpos celestes mais densos, como anãs brancas por exemplo, que apresentam desvios para o vermelho mais intensos e não apresentam os problemas do Sol. Porém, as anãs brancas cujas massas podem ser calculadas facilmente são, em sua maioria, sistemas binários e devido à proximidade entre as estrelas componentes, há um grande espalhamento da radiação emitida. Uma exceção é o par Eridani 40 A e B, que estão suficientemente separadas tal que os efeitos de espalhamento não são tão graves[13]. Entretanto, o problema com esta é que seu 
período de revolução é muito longo e até hoje não se conseguiu determinar a massa de Eridani 40 B com boa acurácia. Alguns cálculos ${ }^{5}$ mostram um desvio $\Delta \nu / \nu=-(7 \pm 1) \cdot 10^{-5}$, o que já está a uma ordem de grandeza melhor em comparação ao desvio apresentado pelo Sol, mas ainda é um resultado insatisfatório.

\subsubsection{Forma matricial da métrica}

O tensor métrico $g_{\mu \nu}$ e a métrica de Minkowski $\eta_{\mu \nu}$ podem ser expressos sob a forma matricial:

$$
g=D^{T} \eta D
$$

Onde D é uma matriz definida por,

$$
\begin{aligned}
& D_{\alpha \mu} \equiv \frac{\partial \xi^{\alpha}}{\partial x^{\mu}} \\
& D_{\mu \alpha}^{T}=D_{\alpha \mu} .
\end{aligned}
$$

E essa matriz por definição não é singular, uma vez que a transformação do referencial da partícula para o referencial do laboratório não apresenta singularidade. Assim, existe uma matriz $D^{-1}$ tal que

$$
\left(D^{-1} D\right)_{\mu \nu}=\frac{\partial x^{\mu}}{\partial \xi^{\alpha}} \frac{\partial \xi^{\alpha}}{\partial x^{\nu}}=\delta_{\nu}^{\mu} \Rightarrow \operatorname{Det}(D) \neq 0
$$

\footnotetext{
${ }^{5}$ Usando a aproximação $g=1+2 \phi$ mostrada na seção anterior, para a Terra e para
} Eridani 40B 
Uma transformação como a do tensor métrico, onde D tem um determinante não nulo, é chamada de congruência. Importante salientar que, embora o tensor métrico se relacione com a métrica de Mikowski por uma congruência, não significa que os autovalores do tensor métrico sejam os mesmos do tensor de Minkowski. Contudo, há um teorema chamado Lei de Sylvester da inércia o qual mostra que o número de autovalores positivos, negativos ou nulos não se altera sob uma transformação de congruência. Ou seja, o tensor métrico deve ter três autovalores positivos (ou negativos), um negativo (ou positivo) e nenhum autovalor nulo e é esta propriedade que distingüe o espaço-tempo de $(3+1)$ dimensões de um espaço de 4 dimensões espaciais, ou de um espaçotempo $(2+2)$ dimensões. 


\subsection{Princípio de Covariância Geral}

Na seção anterior, vimos que o princípio da equivalência nos permite descobrir os efeitos da gravitação num determinado sistema físico e poderíamos continuar com este "ansatz", porém há um método mais elegante e conciso e com mesmo conteúdo físico. Este método é baseado numa forma alternativa do princípio da equivalência e é chamado Princípio da Covariância Geral. Esse princípio diz que na presença de um campo gravitacional arbitrário, a equação de um dado processo físico mantém sua forma se:

(1)- Ela mantém sua forma na ausência de gravidade (i.e. a equação está de acordo com os princípios da relatividade especial).

(2)- A equação é covariante, i.e. ela preserva sua forma sob uma mudança de sistema de coordenadas $x^{\mu} \rightarrow x^{\prime \mu}$.

Para vermos que o princípio de covariância vem do princípio da equivalência, consideremos uma região do espaço com um campo gravitacional arbitrário e que nessa região, haja uma equação física que satisfaz o princípio da covariância Então, de acordo com (2), a equação é verdadeira em qualquer sistema de coordenadas se ela o for em um dado. Considerando um ponto qualquer do espaço de acordo com o princípio da equivalência, podemos escolher um referencial localmente inercial, onde os efeitos de curvatura causados pela gravidade são nulos e a equação mantém sua forma nesse sistema (requisito (1)) e por (2), ela manterá sua forma em todos os demais. 


\subsubsection{Vetores e Tensores}

Vetores e Tensores (ou simplesmente tensores) são objetos que possuem uma lei de transformação bem definida sob uma mudança de coordenadas. $\mathrm{O}$ mais simples deles chama-se escalar ou tensor de ordem 0 , o qual é invariante sob uma mudança de coordenadas.

O próximo em escala de dificuldade ${ }^{6}$, é o tensor de primeira ordem ou vetor, que pode ser contravariante ou covariante.

Um vetor contravariante é o vetor cuja lei de transformação é:

$$
V^{\prime \mu}\left(x^{\prime}\right)=\frac{\partial x^{\prime \mu}}{\partial x^{\nu}} V^{\nu}(x)
$$

E um vetor covariante é o vetor cuja lei de transformação é:

$$
U_{\mu}^{\prime}\left(x^{\prime}\right)=\frac{\partial x^{\nu}}{\partial x^{\prime \mu}} U_{\nu}(x)
$$

Podemos generalizar esses conceitos para tensores de ordem n. Por exemplo:

$$
T_{\rho}^{\prime \mu \nu}\left(x^{\prime}\right)=\frac{\partial x^{\prime \mu}}{\partial x^{\alpha}} \frac{\partial x^{\prime \nu}}{\partial x^{\beta}} \frac{\partial x^{\gamma}}{\partial x^{\prime \rho}} T_{\gamma}^{\alpha \beta}(x),
$$

assim, sem grandes dificuldades, conseguimos verificar que a métrica $g_{\mu \nu}$ também é um tensor, bem como a sua inversa $g^{\mu \nu}$.

\footnotetext{
${ }^{6}$ Há objetos intermédiários entre os escalares e os vetores, chamados de pseudoescalares que são objetos que não seguem a lei de transformação dos vetores, mas que mudam de sinal dependendo do sistema de referência. Um exemplo de pseudo-escalar é o objeto $\epsilon^{i j} \partial_{i} A_{j}$ em $(2+1)$ dimensões.
} 


\subsubsection{Densidades Tensoriais}

Consideremos o determinante do tensor métrico $g \equiv-\operatorname{det}\left(g_{\mu \nu}\right)$. De acordo com o exposto acima, vemos que o tensor métrico num sistema de coordenadas $x^{\prime \mu}$ pode ser escrito de acordo com (2.50):

$$
g_{\mu \nu}^{\prime}=\frac{\partial x^{\rho}}{\partial x^{\prime \mu}} g_{\rho \sigma} \frac{\partial x^{\sigma}}{\partial x^{\prime \nu}}
$$

Calculando o determinante de ambos os lados da equação matricial acima, temos que

$$
g^{\prime}=\left|\frac{\partial x}{\partial x^{\prime}}\right|^{2} g=\left|\frac{\partial x^{\prime}}{\partial x}\right|^{-2} g
$$

onde $\left|\frac{\partial x^{\prime}}{\partial x}\right|$ é o jacobiano da transformação $x^{\mu} \rightarrow x^{\prime \mu}$. Um objeto que se transforma como um escalar, exceto por um fator multiplicativo que é uma potência do jacobiano, é chamado densidade escalar. Um objeto de ordem n que se transforma como um tensor, exceto por um fator multiplicativo que é uma potência do jacobiano é chamado densidade tensorial e o expoente do jacobiano é chamado peso da densidade tensorial. Por exemplo, o determinante da métrica é uma densidade de peso -2. Uma propriedade útil é que dada uma densidade tensorial de peso P, este pode expresso como um tensor ordinário com um fato extra $g^{P / 2}$. A importância desses objetos vem de um teorema do cálculo integral (teorema de Fubini), que diz que sob uma mudança do sistema de coordenadas, o elemento de volume $d^{4} x$ transforma-se 
da seguinte maneira:

$$
d^{4} x^{\prime}=\left\|\frac{\partial x^{\prime}}{\partial x}\right\| d^{4} x=\sqrt{\frac{g}{g^{\prime}}} d^{4} x .
$$

Ou seja, $\sqrt{g} d^{4} x$ é um elemento de volume invariante sob transformações de coordenadas.

\subsubsection{Transformação da Conexão Afim}

Veremos agora que a conexão afim não é um tensor. Para verificarmos isso, seja $\Gamma_{\mu \nu}^{\prime \lambda}$ a conexão afim sob uma mudança do sistema de coordenadas. Partindo de (2.19) e da definição de tensores, veremos que

$$
\Gamma_{\mu \nu}^{\prime \lambda}=\frac{\partial x^{\prime \lambda}}{\partial x^{\rho}} \frac{\partial x^{\sigma}}{\partial x^{\prime \nu}} \frac{\partial x^{\tau}}{\partial x^{\prime \mu}} \Gamma_{\sigma \tau}^{\prime \rho}+\frac{\partial x^{\prime \lambda}}{\partial x^{\rho}} \frac{\partial^{2} x^{\rho}}{\partial x^{\prime \mu} \partial x \nu}
$$

onde o segundo termo à direita da equação acima deveria ser zero se a conexão afim fosse um tensor.

\subsubsection{Derivada Covariante}

Seja $V^{\prime \mu}$ um vetor contravariante, isto é:

$$
V^{\prime \mu}=\frac{\partial x^{\prime \mu}}{\partial x^{\nu}} V^{\nu}
$$

Derivando com respeito a $x^{\prime \lambda}$, temos:

$$
\frac{\partial V^{\prime \mu}}{\partial x^{\prime \lambda}}=\frac{\partial x^{\prime \mu}}{\partial x^{\nu}} \frac{\partial x^{\rho}}{\partial x^{\prime \lambda}} \frac{\partial V^{\nu}}{\partial x^{\rho}}+\frac{\partial^{2} x^{\prime \mu}}{\partial x^{\nu} \partial x^{\rho}} \frac{\partial x^{\rho}}{\partial x^{\lambda}} V^{\nu}
$$


assim, verificamos que este objeto não é um tensor, ou seja derivadas de tensores não são necessariamente tensores. Porém podemos construir um tensor (isto é, um objeto covariante) com a derivada que calculamos. De fato, de (2.54) e de (2.55), temos que:

$$
\frac{\partial V^{\prime \mu}}{\partial x^{\prime \lambda}}+\Gamma_{\lambda \kappa}^{\prime \mu} V^{\prime \kappa}=\frac{\partial x^{\prime \mu}}{\partial x^{\nu}} \frac{\partial x^{\rho}}{\partial x^{\prime \lambda}}\left(\frac{\partial V^{\nu}}{\partial x^{\rho}}+\Gamma_{\rho \sigma}^{\prime \nu} V^{\prime \sigma}\right)
$$

Com isso nós definimos a Derivada covariante, que é representada por $V_{; \lambda}^{\mu}=$ $\frac{\partial V^{\mu}}{\partial x^{\lambda}}+\Gamma_{\lambda \sigma}^{\mu} V^{\sigma}$ e que satisfaz a equação de transformação:

$$
V_{; \lambda}^{\prime \mu} \equiv \frac{\partial x^{\prime \mu}}{\partial x^{\nu}} \frac{\partial x^{\rho}}{\partial x^{\prime \lambda}} V_{; \rho}^{\nu}
$$

A derivada covariante de um tensor $\mathrm{T}$ de ordem $\mathrm{n}$ com relação a $x^{\rho}$ é feita como segue:

1. Calcula-se $\partial T::: / \partial x^{\rho}$;

2. Para cada índice contravariante $\mu$, adiciona-se um termo $\Gamma_{\nu \rho}^{\mu}$, multiplicado por $\mathrm{T}$ com $\mu$ trocado por $\nu$;

3. Para cada índice covariante $\lambda$, subtrai-se um termo $\Gamma_{\lambda \rho}^{\kappa}$, multiplica por T com $\lambda$ trocado por $\kappa$.

Podemos verificar que a derivada covariante de um tensor de ordem n é um tensor de ordem $n+1$. A importância da derivada covariante está em duas de suas propriedades, a saber, ela transforma tensores em outros tensores e ela se reduz à derivada ordinária para uma região sem gravidade. Com 
isso, podemos fazer o seguinte raciocínio para descobrirmos os efeitos da gravitação sobre um dado sistema físico: Escrever as equações do processo físico no referencial localmente inercial, mudar $\eta_{\mu \nu}$ por $g_{\mu \nu}$, e mudar todas as derivadas ordinárias por derivadas covariantes. As equações resultantes serão covariantes e verdadeiras na ausência de gravidade (ou em outras palavras, no referencial localmente inercial) e, de acordo com o princípio da covariância, serão verdadeiras em qualquer referencial com campo gravitacional arbitrário, lembrando que isso vale para pequenas escalas quando comparadas com a escala de variação típica do campo gravitacional.

\subsubsection{Transporte paralelo}

Consideremos um tensor $T(\tau)$, definido apenas sobre a curva $x^{\mu}(\tau)$. Para tensores desse tipo poderíamos falar em diferenciação ao longo da curva na qual o tensor está definido. Por exemplo, consideremos um vetor contravariante $A^{\mu}(\tau)$

$$
\frac{d A^{\prime \mu}(\tau)}{d \tau}=\frac{d}{d \tau}\left(\frac{\partial x^{\prime \mu}}{\partial x^{\nu}} A^{\nu}(\tau)\right)=\frac{\partial x^{\prime \mu}}{\partial x^{\nu}} \frac{d A^{\nu}}{d \tau}+\frac{\partial^{2} x^{\prime \mu}}{\partial x^{\nu} \partial x^{\lambda}} \frac{d x^{\lambda}}{d \tau} A^{\nu}(\tau)
$$

Ou seja, a derivada do vetor com respeito a parâmetro $\tau$ não é um vetor, devido ao termo não homogêneo (segundo termo) apresentado na eq.(2.59), analogamente ao que ocorreu quando escrevemos a transformação da conexão afim. Assim, somos levados novamente a definir uma derivada covariante de 
$A^{\prime \mu}(\tau)$ ao longo da curva $x^{\mu}(\tau)$ por

$$
\frac{D A^{\mu}(\tau)}{D \tau} \equiv \frac{d A^{\mu}}{d \tau}+\frac{\partial^{2} x^{\prime \mu}}{\partial x^{\nu} \partial x^{\lambda}} \frac{d x^{\lambda}}{d \tau} A^{\nu}=\frac{d A^{\mu}}{d \tau}+\Gamma_{\nu \lambda}^{\mu} \frac{d x^{\lambda}}{d \tau} A^{\nu}
$$

De fato, temos que:

$$
\begin{aligned}
& \frac{D A^{\prime}(\tau)}{D \tau}=\frac{d A^{\prime \mu}}{d \tau}+\Gamma_{\nu \lambda}^{\mu} \frac{d x^{\lambda}}{d \tau} A^{\prime \nu} \\
& =\frac{d}{d \tau}\left(\frac{\partial x^{\prime \mu}}{\partial x^{\nu}} A^{\nu}(\tau)\right)+\Gamma_{\nu \lambda}^{\mu} \frac{d x^{\lambda}}{d \tau} \frac{\partial x^{\prime \mu}}{\partial x^{\nu}} A^{\nu}(\tau),
\end{aligned}
$$

que, comparando com a Eq. (2.60):

$$
\frac{D A^{\prime \mu}(\tau)}{D \tau}=\frac{\partial x^{\prime \mu}}{\partial x^{\nu}} \frac{D A^{\nu}}{D \tau}
$$

Ou seja, verificamos que a derivada covariante de um vetor, ao longo de $\tau$, também é um vetor e, analogamente, definimos a derivada covariante de um vetor covariante $B_{\mu}(\tau)$ :

$$
\frac{D B_{\mu}}{D \tau}=\frac{d B_{\mu}}{d \tau}-\Gamma_{\mu \nu}^{\lambda} \frac{d x^{\nu}}{d \tau} B_{\lambda}
$$

De acordo com (2.62) e (2.63), a derivada covariante de um tensor genérico ao longo de uma curva sobre a qual ele está definido pode ser calculada de forma semelhante ao cálculo da derivada covariante geral. Por exemplo, seja um tensor genérico $T_{\cdot \nu}^{\mu}$,

$$
\frac{D T_{\cdot \nu}^{\mu}}{D \tau}=\frac{d T_{\cdot \nu}^{\mu}}{d \tau}+\Gamma_{\lambda \rho}^{\mu} \frac{d x^{\lambda}}{d \tau} T_{\cdot \nu}^{\rho}-\Gamma_{\lambda \nu}^{\sigma} \frac{d x^{\lambda}}{d \tau} T_{\cdot \sigma}^{\mu},
$$


que, fazendo as devidas simplificações, chegamos à expressão anterior em termos da derivada covariante

$$
\frac{D T_{\cdot \nu}^{\prime \mu}}{D \tau}=\frac{\partial x^{\prime} \mu}{\partial x^{\rho}} \frac{\partial x^{\sigma}}{\partial x^{\prime \nu}} \frac{D T_{\cdot \sigma}^{\rho}}{D \tau} .
$$

Ao calcularmos a derivada de um tensor $\mathrm{T}$ ao longo do caminho $\tau$, dizemos que T sofreu um transporte paralelo sobre $\tau$ 


\subsection{Tetradas}

Como já vimos, a métrica é dada por

$$
g_{\mu \nu}(x)=V_{\bullet \mu}^{\alpha}(x) V_{\bullet \nu}^{\beta}(x) \eta_{\alpha \beta},
$$

$\operatorname{com} V_{\bullet \mu}^{\alpha} \equiv \frac{\partial \xi^{\alpha}(x)}{\partial x^{\mu}}$ e verificamos que ao fazermos a mudança $x^{\mu} \rightarrow x^{\prime \mu}$, temos

$$
V_{\bullet \mu}^{\alpha}(x) \rightarrow V_{\bullet \mu}^{\prime \alpha}\left(x^{\prime}\right)=\frac{\partial \xi^{\alpha}\left(x^{\prime}\right)}{\partial x^{\prime \mu}}=\frac{\partial \xi^{\alpha}(x)}{\partial x^{\prime \mu}}=\frac{\partial \xi^{\alpha}(x)}{\partial x^{\nu}} \frac{\partial x^{\nu}}{\partial x^{\prime \mu}}=\frac{\partial x^{\nu}}{\partial x^{\prime \mu}} V_{\bullet \nu}^{\alpha}(x)
$$

Assim, vemos que $V_{\bullet \mu}^{\alpha}$ forma 4 quadrivetores covariantes ao invés de um tensor e este conjunto de vetores covariantes é chamado de tetrada ou vierbein. Podemos entender isso a partir do postulado de Gauss mencionado anteriormente, ou seja, em cada ponto podemos definir um espaço tangente no qual vale a métrica de Minkowski e a relatividade especial. Isso nos permite escrever os espinores num espaço-tempo curvo.

Consideremos agora um vetor contravariante $A^{\mu}(x)$, podemos usar as tetradas para relacionar x ao sistema localmente inercial $\xi_{x}^{\alpha}$ :

$$
A^{\prime \mu}\left(x^{\prime}\right)=\frac{\partial x^{\prime \mu}}{\partial x^{\nu}} A^{\nu}(x)=\frac{\partial x^{\prime \mu}}{\partial \xi^{\alpha}} \frac{\partial \xi^{\alpha}}{\partial x^{\nu}} A^{\nu}(x)=\frac{\partial x^{\prime \mu}}{\partial \xi^{\alpha}} V_{\bullet \nu}^{\alpha} A^{\nu}
$$

e se definirmos

$$
\begin{aligned}
& * A^{\mu} \equiv V_{\bullet \mu}^{\alpha} A^{\mu} \Rightarrow A^{\prime \mu}=\frac{\partial x^{\prime \mu}}{\partial \xi^{\alpha}}\left(* A^{\nu}\right) \\
& B_{\bullet \beta}^{\alpha}=V_{\bullet \mu}^{\alpha} V_{\beta}^{\bullet \nu} B_{\bullet \nu}^{\mu}
\end{aligned}
$$


onde, $V_{\beta}^{\bullet \nu}=\eta_{\alpha \beta} g^{\mu \nu} V_{\bullet \mu}^{\alpha}$, temos:

$$
\begin{aligned}
& g_{\mu \nu}=V_{\bullet \mu}^{\alpha} V_{\bullet \nu}^{\beta} \eta_{\alpha \beta} \Rightarrow g^{\mu \sigma} g_{\mu \nu}=V_{\bullet \mu}^{\alpha} V_{\bullet \nu}^{\beta} \eta_{\alpha \beta} g^{\mu \sigma} \\
& \therefore \delta_{\nu}^{\sigma}=V_{\beta}^{\bullet \sigma} V_{\bullet \nu}^{\beta} .
\end{aligned}
$$

Uma derivada ordinária é certamente um vetor com respeito à transformação geral de coordenadas, no sentido que, sob uma transformação de coordenada $x \rightarrow x^{\prime}$, ela se transforma de acordo com a regra:

$$
\frac{\partial}{\partial x^{\mu}} \rightarrow \frac{\partial}{\partial x^{\prime \mu}}=\frac{\partial x^{\nu}}{\partial x^{\prime \mu}} \frac{\partial}{\partial x^{\nu}}
$$

Se todos os campos que aparecem na ação forem escalares e sabendo que sempre há derivada de campos na ação, não haveria índice contravariante para contrair com o índice covariante $\mu$. Então, para fazer da ação um escalar, é necessário introduzir a tetrada $V_{\alpha}^{\bullet \mu}$

$$
\begin{aligned}
& V_{\alpha}^{\bullet \mu} \frac{\partial}{\partial x^{\mu}} * \psi(x) \rightarrow \Lambda_{\alpha}^{\bullet \beta}(x) V_{\beta}^{\bullet \mu}(x) \frac{\partial}{\partial x^{\mu}}[D(\Lambda) * \psi(x)], \\
& =\Lambda_{\alpha}^{\bullet \beta}(x) V_{\beta}^{\bullet \mu}(x)\left[D(\Lambda) \frac{\partial}{\partial x^{\mu}} * \psi(x)+\left(\frac{\partial}{\partial x^{\mu}} D(\Lambda)\right) * \psi(x)\right],
\end{aligned}
$$

em que $\Lambda_{\alpha}^{\bullet \beta}$ é o elemento da matriz de transformação de Lorentz e $D(\Lambda)$ é uma matriz de representação do grupo de Lorentz. Contudo, o que precisamos é incorporar derivadas na ação sob a forma de um operador, que não só é um escalar como também é um vetor de Lorentz no sentido que, para uma transformação dependente da posição $\Lambda_{\bullet \beta}^{\alpha}(x)$,

$$
\mathcal{D}_{\alpha}(* \psi(x)) \rightarrow \Lambda_{\alpha}^{\bullet \beta}(x) D(\Lambda(x)) \mathcal{D}_{\beta} * \psi(x)
$$


Ansatz: $\mathcal{D}_{\alpha} \equiv V_{\alpha}\left[\frac{\partial}{\partial x^{\mu}}+\Gamma_{\mu}\right], \operatorname{com} \Gamma_{\mu} \equiv \frac{1}{2} \sigma^{\alpha \beta} V_{\alpha}^{\bullet \nu}(x) V_{\beta \nu ; \mu}=V_{\alpha}^{\bullet \mu} \partial_{\mu}-\frac{i}{4} \sigma^{b c} \omega_{b c \mu}$. Onde $\omega_{b c \mu}$ é a chamada conexão de spin e assim, vemos que a ação pode ser escrita, segundo esse formalismo, como:

$$
S=\int d^{4} x \sqrt{g} V_{a}^{\bullet \mu} U_{\bullet \mu}^{a}
$$

onde $U_{\bullet \mu}^{a}$ em geral contém derivadas e pode ser entendido como a lagrangeana neste formalismo.

Como um exemplo, vejamos a equação de Dirac num espaço-tempo curvo. Considerando a equação de Dirac no sistema de coordenadas local $\xi^{a}$ :

$$
\gamma^{a} \partial_{a} \psi+i m \psi=0
$$

e como vimos, a derivada covariante muda, assim como as matrizes de Dirac, devido à dificuldade na representação dos espinores num espaço curvo. Por isso, faz-se necessário o uso das tetradas, ou seja, substituimos $\partial_{a}$ por $V_{a}^{\bullet \mu} \mathcal{D}_{\mu}$, onde $\mathcal{D}_{\mu}$ é a derivada covariante com respeito ao sistema $x^{\mu}$. Assim, chegamos à Equação de Dirac num espaço-tempo curvo

$$
\gamma^{a} V_{\bullet a}^{\mu} \mathcal{D}_{\mu} \Psi+i m \psi=0
$$

Por estarmos num espaço curvo, usamos a tetrada para podermos representar os espinores e a derivada covariante também é modificada, sendo escrita como:

$$
\mathcal{D}_{\mu} \doteq \partial_{\mu}-\frac{i}{4} \eta_{a c} \omega_{b \mu}^{c} \sigma^{a b}
$$


em que $\sigma^{a b}$ é o comutador das matrizes de Dirac e $\omega_{b \mu}^{c}$ é a chamada conexão de spin, definida como:

$$
\omega_{b \mu}^{c} \doteq V_{\bullet \nu}^{c} \partial_{\mu} V_{\bullet b}^{\nu}+V_{\bullet \nu}^{c} V_{b}^{\bullet \sigma} \Gamma_{\sigma \mu}^{\nu}
$$

mantendo assim a mesma estrutura da equação do espaço-tempo plano. 


\section{Capítulo 3}

\section{Férmions num Campo de Calibre}

\subsection{O Campo de Calibre de Chern-Simons}

Como sabemos, a teoria de Maxwell é definida por meio do campo fundamental $A_{\mu}$, sendo a sua lagrangiana

$$
\mathcal{L}_{M}=-\frac{1}{4} F_{\mu \nu} F^{\mu \nu}-A_{\mu} J^{\mu}
$$

expressa por meio do tensor de campo eletromagnético (também chamado de curvatura em analogia à teoria da Gravitação) $F_{\mu \nu} \equiv \partial_{\mu} A_{\nu}-\partial_{\nu} A_{\mu}$ e pela corrente de matéria $J^{\mu}$, que é conservada. A lagrangiana de Maxwell, como já sabemos, é invariante por transformações de calibre dos campos $A_{\mu} \rightarrow A_{\mu}+\partial_{\mu} \Lambda$ e conseqüentemente as equações de movimento

$$
\partial_{\mu} F^{\mu \nu}=J^{\nu}
$$


também o serão.

Como podemos verificar facilmente, a teoria de Maxwell pode ser definida em um espaço de dimensão arbitrária, apenas modificando o alcance do índice do campo básico $A_{\mu}$ indo de 0 a (d-1). Num espaço-tempo d-dimensional, e essa mudança não muda a forma da lagrangiana tampouco das equações de movimento, mudando apenas o numero de campos básicos que há para determinado espaço-tempo ${ }^{1}$. Assim, vemos que a mudança mais significativa é devida ao campo magnético comportar-se como um pseudoescalar $B=\epsilon^{i j} \partial_{i} A_{j}$ num espço-tempo tridimensional e não existe num espaço-tempo bidimensional.

Uma teoria de calibre que apresenta algumas diferenças com relação à teoria de Maxwell num espaço-tempo de $(2+1)$ D é a teoria de Chern-Simons, que é descrita pela lagrangiana

$$
\mathcal{L}_{C S}=\frac{\sigma}{2} \epsilon^{\mu \nu \rho} A_{\mu} \partial_{\nu} A_{\rho}-A_{\mu} J^{\mu}
$$

Há vários comentários a se fazer a respeito desta Lagrangiana. Primeiro, não é uma lagrangiana invariante de calibre pois ela envolve o próprio campo $A_{\mu}$ ao passo que a teoria de Maxwell envolve apenas o tensor de campo $F_{\mu \nu}$. Contudo, sob uma transformação de calibre, a lagrangiana de ChernSimons muda apenas por uma derivada total, ou seja, se ignorarmos termos

\footnotetext{
${ }^{1}$ como o tensor de campo é antisimétrico, o número de campos básicos independentes num espaço d-dimensional será $\frac{1}{2} d(d-1)$
} 
de borda, a ação de Chern-Simons preserva a invariância de calibre. Segundo, a lagrangiana de Chern-Simons tem derivadas espaço-temporais de primeira ordem, no lugar de um d'alembertiano como na teoria de Maxwell. Essas duas características nos mostram que a teoria de Chern-Simons apresenta diferenças significativas com relação à teoria de Maxwell. A principal delas podemos ver nas equações de movimento que seguem de (3.3)

$$
\begin{aligned}
& \rho=\sigma B \\
& J^{i}=\sigma \epsilon^{i j} E_{j} .
\end{aligned}
$$

A primeira equação nos diz que o campo mangético é localmente proporcional à densidade de carga e a segunda que o campo elétrico é localmente proporcional à densidade de corrente, o que é uma diferença drástica com relação ao modelo de Maxwell.

\subsection{Propagador de Maxwell-Chern-Simons}

${ }^{2}$ Como já é conhecido da lagrangiana de Maxwell, o cálculo do propagador requer a introdução de um termo de fixação de calibre para que o propagador seja determinado de maneira unívoca. Consideremos agora a lagrangiana de Maxwell-Chern-Simons com um termo de fixação de calibre do

\footnotetext{
${ }^{2}$ Não é necessária a leitura desta seção para entendimento do trabalho. Trata-se apenas de uma ilustração.
} 


$$
\begin{aligned}
& \text { tipo }-\frac{1}{2 \xi e^{2}}\left(\partial_{\mu} A^{\mu}\right)^{2} \\
& \mathcal{L}_{M C S}=-\frac{1}{4 e^{2}} F_{\mu \nu} F^{\mu \nu}+\frac{\sigma}{2} \epsilon^{\mu \nu \rho} A_{\mu} \partial_{\nu} A_{\rho}-\frac{1}{2 \xi e^{2}}\left(\partial_{\mu} A^{\mu}\right)^{2} \\
& S=\int d^{4} x\left[-\frac{1}{2 e^{2}}\left(\partial_{\mu} A_{\nu} \partial^{\mu} A^{\nu}-\partial_{\mu} A_{\nu} \partial^{\nu} A^{\mu}\right)\right]+\frac{\sigma}{2} \epsilon^{\mu \nu \rho} A_{\mu} \partial_{\nu} A_{\rho}-\frac{1}{2 \xi e^{2}} \partial_{\mu} A^{\mu} \partial_{\nu} A^{\nu}
\end{aligned}
$$

A ação pode ser calculada por integração por partes, desprezando os termos de superfície

$$
\begin{aligned}
& S=\int d^{4} x \frac{1}{2 e^{2}}\left(A^{\mu} g_{\mu \nu} \square-A^{\nu}-A^{\mu} \partial_{\nu} \partial_{\mu} A^{\nu}\right)+\frac{\sigma}{2} \epsilon_{\mu \nu \rho} A^{\mu} \partial^{\rho} A^{\nu}-\frac{1}{2 \xi e^{2}} A^{\mu} \partial^{\mu} \partial_{\nu} A^{\nu} \\
& =\int d^{4} x A^{\mu}\left[\frac{1}{2 e^{2}}\left(g_{\mu \nu} \square-\partial_{\nu} \partial_{\mu}\right)+\frac{\sigma}{2} \epsilon_{\mu \rho \nu} \partial^{\rho}+\frac{1}{2 \xi e^{2}} \partial_{\mu} \partial_{\nu}\right] A^{\nu}
\end{aligned}
$$

lembrando que o termo entre colchetes é o núcleo da ação e o propagador, que é uma função de Green, pode ser calculado pela identidade:

$$
\begin{aligned}
& \mathcal{O}_{\mu \nu}(x) \Delta^{\nu \alpha}(x-y)=i \delta_{\mu}^{\alpha} \delta^{3}(x-y) \\
& \left(\frac{g_{\mu \nu} \square}{e^{2}}-\frac{\partial_{\mu} \partial_{\nu}}{e^{2}}+\sigma \epsilon_{\mu \rho \nu} \partial^{\rho}+\frac{1}{2 \xi e^{2}} \partial_{\mu} \partial_{\nu}\right) \Delta^{\nu \alpha}(x-y)=i \delta_{\mu}^{\alpha} \delta^{3}(x-y)(3.9
\end{aligned}
$$

Aplicando a transformada de Fourier sobre o propagdor

$$
\Delta^{\nu \alpha}(x-y)=\int(d k) \Delta^{\nu \alpha}(k) e^{i k(x-y)}
$$

ou seja,

$$
\partial_{\mu} \Delta^{\nu \alpha}(x-y)=\int(d k) i k_{\mu} \Delta^{\nu \alpha}(k) e^{i k(x-y)}
$$

Assim, escrevemos a expressão 3.9 no espaço dos momentos:

$$
\int(d k) e^{i k(x-y)}\left(-\frac{g_{\mu \nu} k^{2}}{e^{2}}+\frac{k_{\mu} k_{\nu}}{e^{2}}+i \sigma \epsilon_{\mu \rho \nu} k^{\rho}-\frac{1}{2 \xi e^{2}} k_{\mu} k_{\nu}\right) \Delta^{\nu \alpha}(k)=i \delta_{\mu}^{\alpha} \int(d k) e^{i k(x-y)}
$$


e como as integrais são iguais, os integrandos também são, portanto

$$
\left(-\frac{g_{\mu \nu} k^{2}}{e^{2}}+\frac{k_{\mu} k_{\nu}}{e^{2}}+i \sigma \epsilon_{\mu \rho \nu} k^{\rho}-\frac{1}{2 \xi e^{2}} k_{\mu} k_{\nu}\right) \Delta^{\nu \alpha}(k)=i \delta_{\mu}^{\alpha} .
$$

Agora, para o cálculo do propagador, usaremos um ansatz geral, isto é,

$$
\begin{aligned}
& \Delta^{\nu \alpha}(k)=A g^{\nu \alpha}+B k^{\nu} k^{\alpha}+C \epsilon^{\nu \lambda \alpha} k_{\lambda}, \\
& \left(-\frac{g_{\mu \nu} k^{2}}{e^{2}}+\frac{k_{\mu} k_{\nu}}{e^{2}}+i \sigma \epsilon_{\mu \rho \nu} k^{\rho}-\frac{1}{2 \xi e^{2}} k_{\mu} k_{\nu}\right) \cdot\left(A g^{\nu \alpha}+B k^{\nu} k^{\alpha}+C \epsilon^{\nu \lambda \alpha} k_{\lambda}\right)=i \delta_{\mu}^{\alpha} \\
& -\frac{A k^{2}}{e^{2}} \delta_{\mu}^{\alpha}-\frac{B k^{2} k_{\mu} k^{\alpha}}{e^{2}}-\frac{C k^{2} k_{\lambda} g_{\mu \nu} \epsilon^{\nu \lambda \alpha}}{e^{2}} \\
& +\left(1-\frac{1}{2 \xi}\right)\left[\frac{A k_{\mu} k^{\alpha}}{e^{2}}+\frac{B k^{2} k_{\mu} k^{\alpha}}{e^{2}}+\frac{C \epsilon^{\nu \lambda \alpha} k_{\mu} k_{\nu} k_{\lambda}}{e^{2}}\right]+i \sigma A g^{\nu \alpha} \epsilon_{\mu \rho \nu} k^{\rho}+i \sigma B k^{\nu} k^{\alpha} k^{\rho} \epsilon_{\mu \rho \nu} \\
& +i \sigma C \epsilon^{\nu \lambda \alpha} \epsilon_{\mu \rho \nu} k_{\lambda} k^{\rho}=i \delta_{\mu}^{\alpha} .
\end{aligned}
$$

Com isso chegamos a três equações e três incógnitas, a saber,

$$
\begin{aligned}
& -\frac{A k^{2}}{e^{2}}-i \sigma C k^{2}=i \\
& \left(-\frac{B k^{2}}{2 \xi e^{2}}+\frac{\left(1-\frac{1}{2 \xi}\right) A}{e^{2}}+i \sigma C\right) k_{\mu} k^{\alpha}=0, \\
& \left(i \sigma A-\frac{k^{2} C}{e^{2}}\right) g_{\mu \nu} \epsilon^{\nu \lambda \alpha} k_{\lambda}=0 .
\end{aligned}
$$

O sistema acima pode ser resolvido por substituição

$$
\begin{aligned}
& A=-\frac{i e^{2}}{k^{2}}-i \sigma e^{2} C \\
& -i \sigma\left(\frac{i e^{2}}{k^{2}}+i e^{2} \sigma C\right)-\frac{k^{2} C}{e^{2}}=0 \\
& C\left(e^{2} \sigma^{2}-\frac{k^{2}}{e^{2}}\right)+\frac{e^{2} \sigma}{k^{2}}=0 \\
& C\left(\frac{k^{2}}{e^{2}}-e^{2} \sigma\right)=\frac{e^{2} \sigma}{k^{2}} \\
& C=\frac{\sigma\left(e^{4}\right)}{k^{2}\left(k^{2}-\left(\sigma e^{2}\right)^{2}\right)} .
\end{aligned}
$$


Substituindo nas equações anteriores, achamos os valores de A e B,

$$
\begin{aligned}
& A=-\frac{i e^{2} k^{2}}{\left.k^{2}\left[k^{2}-\left(\sigma e^{2}\right)^{2}\right)\right]}, \\
& B=\frac{i e^{4}}{k^{2}\left[k^{2}-\left(\sigma e^{2}\right)^{2}\right]}-\frac{2 i \xi e^{2}}{k^{4}},
\end{aligned}
$$

chegando portanto à expressão do propagador no espaço dos momentos:

$$
\Delta^{\nu \alpha}(k)=-i e^{2}\left[\frac{k^{2} g^{\nu \alpha}-e^{2} k^{\nu} k^{\alpha}+i \sigma e^{2} \epsilon^{\nu \lambda \alpha} k_{\lambda}}{k^{2}\left[k^{2}-\left(\sigma e^{2}\right)^{2}\right]}+\frac{2 \xi k^{\nu} k^{\alpha}}{k^{4}}\right] .
$$

$\mathrm{Na}$ expressão do propagador, vemos que ele possui um pólo em $k^{2}=\left(\sigma e^{2}\right)^{2}$ semelhantemente ao que ocorre para campos massivos. Por isso, o modelo de MCS é por vezes chamado de "topologicamente massivo", com a massa topológica igual a $\sigma e^{2}$. 
3.3. INDUÇÃO DE CHERN-SIMONS EM (2+1)D NA TEORIA DE CALIBRE39

\subsection{Indução de Chern-Simons em $(2+1) \mathrm{D}$ na}

\section{Teoria de Calibre}

\subsubsection{Informações úteis}

O símbolo $t r_{D}$ indica o traço sobre as matrizes de Dirac e o símbolo tr indica o traço sobre os índices internos do grupo de Lie.

$$
\int(d k) \doteq \int \frac{d^{3} k}{(2 \pi)^{3}}
$$

Integrações sobre o parâmetro s:

1. $\int_{0}^{\infty} \frac{d s}{s^{1 / 2}} e^{-m^{2} s}=\frac{\pi^{1 / 2}}{|m|}$

2. $\int d s s^{1 / 2} e^{-m^{2} s}=\frac{\pi^{1 / 2}}{2|m|^{3}}$. 
Integrais no espaço dos momentos: $:^{3}$

1. $\int(d k) e^{k^{2}}=\frac{1}{8 \pi^{3 / 2}}$

2. $\int(d k) k^{\mu} k^{\nu} e^{k^{2}}=-\frac{g^{\mu \nu}}{16 \pi^{3 / 2}}$.

Escolheremos a métrica como $g^{\mu \nu} \equiv \operatorname{diag}(1,-1,-1)$ e lembrando que as matrizes de Dirac devem satisfazer a álgebra: $\left\{\gamma^{\mu}, \gamma^{\nu}\right\} \equiv 2 g^{\mu \nu}$. Assim, escolhemo-las como

$\gamma^{\mathbf{0}} \equiv \sigma^{2}=\left[\begin{array}{cc}0 & -i \\ i & 0\end{array}\right], \quad \gamma^{\mathbf{1}} \equiv \mathbf{i} \sigma^{\mathbf{1}}=\left[\begin{array}{cc}0 & i \\ i & 0\end{array}\right], \quad \gamma^{\mathbf{2}} \equiv \mathbf{i} \sigma^{\mathbf{3}}=\left[\begin{array}{cc}i & 0 \\ 0 & -i\end{array}\right]$

Em conseqüência disto, temos que:

1. $\operatorname{tr}\left(\gamma^{\mu} \gamma^{\nu} \gamma^{\rho}\right)=2 i \epsilon^{\mu \nu \rho}$

2. $\gamma^{\mu} \gamma^{\nu}=2 g^{\mu \nu}-\gamma^{\nu} \gamma^{\mu}$

3. $\gamma^{\mu} \gamma^{\sigma} \gamma_{\mu}=-\gamma^{\sigma}$

Consideremos agora o sistema de um campo fermiônico acoplado a um campo de calibre externo. A lagrangiana de interação é dada por

$$
\mathcal{L}=\bar{\psi}(\not D-m) \psi=\bar{\psi}\left[\gamma^{\mu}\left(i \partial_{\mu}-e A_{\mu}\right)-m\right] \psi
$$

A ação efetiva $\Gamma_{\text {eff }}$ em 1 laço é definida por [2]:

$$
e^{i \Gamma_{e f f}(A)}=\mathcal{N} \int D \bar{\psi} D \psi e^{i \int \bar{\psi}(\not \supset-m) \psi d^{3} x}
$$

\footnotetext{
${ }^{3}$ Vide apêndice $\mathrm{E}$
} 


\subsection{INDUÇÃO DE CHERN-SIMONS EM $(2+1) D$ NA TEORIA DE CALIBRE41}

onde $\mathcal{N}$ é uma constante de normalização a ser escolhida de modo que $\Gamma_{e f f}(A=0)=0$ e $\not D \doteq \gamma^{\mu} D_{\mu}$ é a derivada covariante, $D_{\mu}=\partial_{\mu}-i e A_{\mu}$. Assim, integrando nos campos fermiônicos (variáveis de Grassmann ${ }^{4}$ ), temos:

$$
e^{i \Gamma_{e f f}}=\mathcal{N} \operatorname{det}(\not D-m)
$$

isto é:

$$
\Gamma_{e f f}=-i \ln [\mathcal{N} \operatorname{det}(\not D-m)]=-i \mathcal{I} r[\ln (i \not \partial-e \not A-m)-\ln (i \not \partial-m)](.3 .30)
$$

Na expressão acima, $\mathcal{T} r$ representa o traço sobre as matrizes de Dirac ${ }^{5}$, sobre os índices do grupo de Lie de calibre e sobre a integração no espaço coordenado. Desenvolvendo a expressão acima, temos

$$
\begin{aligned}
& \Gamma_{e f f}=-i \mathcal{T} r \ln \left[\frac{i \not \partial-e \not A-m}{i \not \partial-m}\right] \\
& -i \mathcal{T} r\left\{\ln \left[\frac{i \not \partial-e \not A-m}{i \not \partial-m}\right]+\ln \left[\frac{-i \not \partial-m}{-i \not \partial-m}\right]\right\}, \\
& \Gamma_{e f f}=-i \mathcal{T} r\{\ln [(i \not \partial-e \not A-m)(-i \not \partial-m)]-\ln [(i \not \partial-m)(-i \not \partial-m)]\}, \\
& =-i \mathcal{T} r\left\{\ln \left[\square+i e \not A \not \partial+e m \not A+m^{2}\right]-\ln [(i \not \partial-m)(-i \not \partial-m)]\right\} . \quad(3.31)
\end{aligned}
$$

Uma solução para (3.31) pode ser escrita sob a forma de uma integral sobre um parâmetro S:

$$
\Gamma_{e f f}^{\prime}=-i \mathcal{T} r\left[\int_{0}^{\infty} \frac{d s}{s} \exp [-s(\square+i e \not A \not \partial-e m \not A)] e^{-m^{2} s}+c t e\right] .
$$

\footnotetext{
${ }^{4}$ vide apêndice $\mathrm{A}$

${ }^{5}$ vide apêndice B para demonstração da identidade acima
} 
Usualmente, S é chamado de tempo próprio de Schwinger por analogia com um desenvolvimento semelhante feito por Schwinger [12] para calcular o propagador do elétron em presença de um campo externo $A^{\mu}(x)$.

Lembrando que o traço de um operador é dado por:

$$
\left.\mathcal{T} r \mathcal{O} \doteq \operatorname{tr}_{D} \operatorname{tr} \int d^{3} x\left\langle x|\mathcal{O}| x^{\prime}\right\rangle\right|_{x=x^{\prime}}
$$

Aplicaremos isso à equação $(3.32)^{6}$, obtendo

$$
\begin{aligned}
& \Gamma_{e f f}^{\prime}=-i \operatorname{Tr}\left\{\int d ^ { 3 } x \int ( d k ) \int _ { 0 } ^ { \infty } \frac { d s } { s } \operatorname { e x p } \left[-s\left[\left(\partial_{\mu}+i k_{\mu}\right)\left(\partial^{\mu}+i k^{\mu}\right)\right.\right.\right. \\
& \left.\left.+i e \not A(\not \partial+i \not k)+e m \not A+m^{2}\right]\right] 1 \\
& \Gamma_{e f f}^{\prime}=-i \operatorname{Tr} \int d^{3} x \int(d k) \int_{0}^{\infty} \frac{d s}{s} e^{-s m^{2}} e^{s k^{2}} \\
& \exp [-s(\square+2 i k . \partial+i e \not A \not \partial-e \not A \not k+e m \not A)] 1 .
\end{aligned}
$$

O que faremos agora é expandir a exponencial acima até terceira ordem para obtermos os termos induzidos de Chern-Simons.

\section{Desenvolvimento do expoente de (3.34)}

$$
\begin{aligned}
& 1-s(\square+2 i k . \partial+i e \not A \not \partial-e \not A \not k+e m \not A) 1 \\
& +\frac{s^{2}}{2}(\square+2 i k . \partial+i e \not A \not \partial-e \not A \not k+e m \not A)(\square+2 i k . \partial+i e \not A \not \partial-e \not A \not k+e m \not A) 1 \\
& -\frac{s^{3}}{3 !}(\square+2 i k . \partial+i e \not A \not \partial-e \not A \not k+e m \not A) . \\
& (\square+2 i k . \partial+i e \not A \not \partial-e \not A \not k+e m \not A)(\square+2 i k . \partial+i e \not A \not \partial-e \not A \not k+e m \not A)+\ldots
\end{aligned}
$$

\footnotetext{
${ }^{6}$ vide apêndice $\mathrm{C}$
} 


\subsection{INDUÇÃO DE CHERN-SIMONS EM $(2+1) D$ NA TEORIA DE CALIBRE43}

Em primeira ordem, os termos anulam-se pois têm derivadas aplicadas ao operador 1. Assim, para segunda ordem, temos:

$$
\frac{s^{2}}{2}(\square+2 i k . \partial+i e \not A \not \partial-e \not A \not k+e m \not A)(\not A)(m-\not k) e .1 .
$$

Eliminando os termos ímpares em k e os que nao dão contribuições para o termo bilinear de Chern-Simons da expressão acima, temos:

$$
\frac{s^{2}}{2}\left(i e^{2} m \not A \not \supset \not A\right)
$$

E em terceira ordem, temos:

$$
\begin{aligned}
& -\frac{s^{3}}{3}(\square+2 i k . \partial+i e \not A \not D-e \not A \not k+e m \not A)\left(2 i m e k^{\mu}\left(\partial_{\mu} \not A\right)-\right. \\
& 2 i e k^{\mu}\left(\partial_{\mu} \not A\right) \not k+i e^{2} m \not A \not D \not A-i e^{2} \not A(\not D \not A) \not k- \\
& \left.e^{2} \not A \not k \not A m+e^{2} \not A \not k \not A \not k+e^{2} m^{2} \not A \not A-e^{2} m \not A \not A \not k\right) .1
\end{aligned}
$$

e obtemos os seguintes termos do tipo Chern-Simons:

$$
-\frac{s^{3}}{3} e^{3} m\left(\not A \not K \not A \not k \not A+\not A \not k \not A \not A \not k+\not A \not A \not k \not A \not k+m^{2} \not A \not A \not A\right)
$$




\section{Cálculo do termo bilinear}

Inserindo (3.37) na expressão da ação efetiva (Eq.(3.32)), temos:

$$
\begin{aligned}
& \Gamma_{e f f}^{(2)}=e^{2} m \int d^{3} x \operatorname{Tr} \int_{0}^{\infty} \frac{d s}{s} \int(d k) \not A \not D \not A \frac{s^{2}}{2} e^{s\left(k^{2}-m^{2}\right)}
\end{aligned}
$$

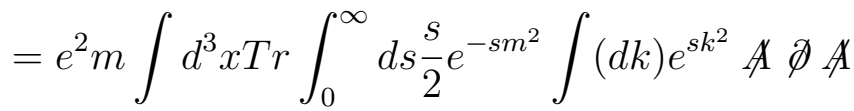

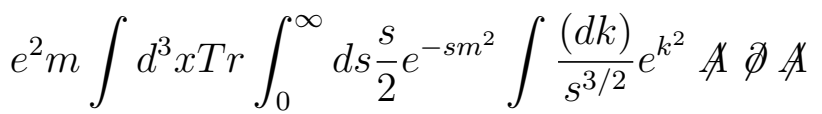

$$
\begin{aligned}
& =e^{2} m \int d^{3} x \operatorname{Tr} \int_{0}^{\infty} \frac{d s}{2 s^{1 / 2}} e^{-s m^{2}} \cdot \frac{1}{8 \pi^{3 / 2}} \not \subset \not D \not A \\
& =\frac{e^{2}}{16 \pi} \frac{m}{|m|} \int d^{3} x \operatorname{trtr}_{D}\left(\gamma^{\mu} \gamma^{\nu} \gamma^{\rho}\right) A_{\mu} \partial_{\nu} A_{\rho} \\
& =\frac{i e^{2}}{8 \pi} \frac{m}{|m|} \epsilon^{\mu \nu \rho} \int d^{3} x \operatorname{tr}\left(A_{\mu} \partial_{\nu} A_{\rho}\right) \text {. }
\end{aligned}
$$

Onde utilizamos as integrais dadas no início do capítulo para s e k.

\section{Cálculo do termo trilinear}

Os termos trilineares são dados pela (3.39) aplicados à definição da ação efetiva (3.32). Faremos o càlculo explícito do primeiro termo

$$
\begin{aligned}
& \Gamma_{e f f(a)}^{(3)} \frac{i e^{3} m^{3}}{3 !} \operatorname{tr}_{D} \operatorname{tr} \int d^{3} x \int_{0}^{\infty} \frac{d s}{s} \int(d k) \not A \not A \not s^{3} e^{s\left(k^{2}-m^{2}\right)} \\
& =\frac{i e^{3} m^{3}}{3 !} \operatorname{trtr}_{D} \int d^{3} x \int_{0}^{\infty} d s s^{2} e^{-s m^{2}} \int(d k) e^{s k^{2}} \not A \not A \not A \\
& =\frac{i e^{3} m^{3}}{3 !} \operatorname{tr}_{D} \operatorname{tr} \int d^{3} x \int_{0}^{\infty} d s s^{2} e^{-s m^{2}} \int \frac{(d k)}{s^{3 / 2}} e^{k^{2}} \not A \not A D \\
& =\frac{i e^{3} m^{3}}{3 !} \operatorname{tr}_{D} \operatorname{tr} \int d^{3} x \int_{0}^{\infty} d s s^{1 / 2} e^{-s m^{2}}\left(\gamma^{\mu} \gamma^{\nu} \gamma^{\rho}\right) A_{\mu} A_{\nu} A_{\rho} \frac{1}{8 \pi^{3 / 2}} \\
& =\frac{i e^{3} m^{3}}{3 !} \operatorname{tr}_{D} \operatorname{tr} \int d^{3} x\left(2 i \epsilon^{\mu \nu \rho} A_{\mu} A_{\nu} A_{\rho} \frac{\sqrt{p i}}{16|m|^{3} \pi^{3 / 2}}\right) \\
& \frac{i e^{3} m^{3}}{48|m|^{3} \pi} i \epsilon^{\mu \nu \rho} \operatorname{tr} \int d^{3} x A_{\mu} A_{\nu} A_{\rho}=\frac{-e^{3}}{48 \pi} \frac{m}{|m|} \epsilon^{\mu \nu \rho} \int d^{3} x A_{\mu} A_{\nu} A_{\rho} \text {. }
\end{aligned}
$$




\subsection{INDUÇÃO DE CHERN-SIMONS EM $(2+1) D$ NA TEORIA DE CALIBRE45}

Calculando de forma análoga os demais termos, chegamos à parte trilinear da ação efetiva

$$
\Gamma^{(3)}=\frac{-e^{3}}{12 \pi} \frac{m}{|m|} \epsilon^{\mu \nu \rho} \int d^{3} x A_{\mu} A_{\nu} A_{\rho}
$$

que combinando com o termo bilinear, nos dá a expressão da ação efetiva

$$
\Gamma_{\text {eff }}=\frac{1}{8 \pi} \frac{m}{|m|} \epsilon^{\mu \nu \rho} \operatorname{tr} \int d^{3} x\left(i e^{2} A_{\mu} \partial_{\nu} A_{\rho}-\frac{2 e^{3}}{3} A_{\mu} A_{\nu} A_{\rho}\right),
$$

como obtida em [16]. Isto nos indica que a interação de férmions com um campo de calibre num espaço bidimensional gera termos do tipo ChernSimons e portanto os quanta do campo de calibre podem ter massa. 


\title{
Capítulo 4
}

\section{Indução de Chern-Simons em}

\author{
$(3+1) \mathrm{D}$
}

\subsection{Teoria de Calibre}

\subsubsection{Convenções Adotadas}

1. $\left(\partial_{\alpha} A\right)$ indica que a derivada age somente sobre o argumento A.

2. O elemento de volume no espaço dos momentos, i.e. $\frac{d^{4} k}{(2 \pi)^{4}}$ será representado por $(\mathrm{dk})$.

3. O produto interno entre dois vetores a e b é representado por $(\mathrm{a}, \mathrm{b})$.

4. A métrica é escolhida como $\eta^{\mu \nu} \doteq \operatorname{diag}(1,-1,-1,-1)$. 
5. Seja $\mathcal{O}$ um operador. O traço sobre o espaço coordenado desse operador, é definido por: $\left.\operatorname{tr}(\mathcal{O}) \doteq \int d^{4} x\left\langle x|\mathcal{O}| x^{\prime}\right\rangle\right|_{x=x^{\prime}}=\left.\int d^{4} x \mathcal{O} \delta^{4}\left(x-x^{\prime}\right)\right|_{x=x^{\prime}}$.

6. Algumas integrais úteis ${ }^{1}$ :

$$
\begin{aligned}
& \int(d k) e^{s k^{2}}=\frac{i}{16 \pi^{2} s^{2}} \\
& \int(d k) e^{s k^{2}} k_{\mu} k_{\nu}=\frac{-i}{32 \pi^{2} s^{3}} \eta_{\mu \nu} \\
& \int(d k) e^{s k^{2}} k_{\mu} k_{\nu} k_{\lambda} k_{\rho}=\frac{i}{64 \pi^{2} s^{4}}\left[\eta_{\mu \nu} \eta_{\lambda \rho}+\eta_{\mu \lambda} \eta_{\nu \rho}+\eta_{\mu \rho} \eta_{\lambda \nu}\right] \\
& \int_{0}^{\infty} d s e^{-s m^{2}} s^{z-1}=\frac{\Gamma(z)}{m^{2 z}}
\end{aligned}
$$

7. As matrizes de Dirac têm a seguinte representação

$$
\gamma^{0}=\left[\begin{array}{cc}
\sigma^{0} & 0 \\
0 & -\sigma^{0}
\end{array}\right], \quad \gamma^{\mathbf{j}}=\left[\begin{array}{cc}
0 & \sigma^{j} \\
-\sigma^{j} & 0
\end{array}\right]
$$

8. A matriz $\gamma^{5}$ é tal que $\left\{\gamma^{\mu}, \gamma^{5}\right\}=0$.

9. Índices referentes ao espaço tangente são representados por letras latinas minúsculas e os índices referentes ao espaço-tempo são representados por letras gregas minúsculas.

\subsubsection{Cálculo do Termo Induzido}

O ponto de partida do nosso estudo é o problema de férmions acoplados com um campo de calibre e também com um campo de fundo $b_{\mu} \gamma^{5}$ que quebra

\footnotetext{
${ }^{1}$ Para o cálculo explícito das tres primeiras integrais, vide apêndice $\mathrm{E}$
} 
a simetria de Lorentz. Assim, a lagrangiana é dada por:

$$
\mathcal{L}=\bar{\psi}\left(\not D-m-\not b \gamma^{5}\right) \psi=\bar{\psi}\left[\gamma^{\mu}\left(i \partial_{\mu}-e A_{\mu}-b_{\mu} \gamma^{5}\right)-m\right] \psi
$$

e aqui $A_{\mu}=A_{\mu}^{a} T^{a}$, onde $T^{a}$ são os geradores de algum grupo de Lie, satisfazendo as relações $\operatorname{tr}\left(T^{a} T^{b}\right)=\delta^{a b} \mathrm{e}\left[T^{a}, T^{b}\right]=i f^{a b c} T^{c}$.

Antes de prosseguirmos com os cálculos, discutiremos brevemente sobre a violação da simetria de Lorentz, induzida pela presença de um campo vetorial clássico (campo de fundo ou éter), permeando todo o espaço. A existência de tal campo daria uma direção preferencial, quebrando a isotropia do espaço tempo proposta na bem sucedida relatividade restrita. Esta idéia ganhou uma atenção especial devido ao fato de que, em um processo de transição de fase, é natural que apareça um campo escalar de fundo não-nulo resultante quando o sistema físico atinge o estado de mínima energia. No caso da simetria de Lorentz, esse valor esperado seria de um campo vetorial ou tensorial, como proposto por Alan Kostelecky [1]. No contexto do modelo padrão das partículas elementares esta quebra explicaria o surgimento de massa nas partículas elementares [11]. Atualmente, há varios trabalhos propondo a quebra da simetria de Lorentz, introduzindo um campo de fundo, sob a forma de um quadrivetor constante no espaço, na lagrangiana de interação (quebra da simetria de Lorentz no contexto da Teoria de Campos). Este campo de fundo seria proveniente da quebra de alguma simetria interna do sistema [1, 4]. Voltando ao nosso cálculo, como o termo induzido de Chern-Simons vem da 
ação efetiva na aproximação de um laço, definamos $\Gamma_{\text {eff }}$ como

$$
\begin{array}{r}
e^{i \Gamma_{e f f}} \doteq \int D \bar{\psi} D \psi \exp \left\{i \int \bar{\psi}\left(\not D-m-\not b \gamma^{5}\right) \psi d^{4} x\right\} \\
\Gamma_{e f f}=-i \ln \left[\mathcal{N} \operatorname{det}\left(\not D-m-\not b \gamma^{5}\right)\right]=-i \mathcal{N} \mathcal{T} r \ln \left(\not D-m-\not b \gamma^{5}\right),
\end{array}
$$

onde $\mathcal{T} r \doteq t r_{D} t r_{\text {coord }} t r_{\text {Lie }}$ indica o traço sobre as matrizes de Dirac, sobre os índices do grupo bem como o traço sobre o espaço coordenado e dos momentos e $\mathcal{N}$ é uma constante de normalização escolhida de tal modo que $\Gamma_{e f f}(A=0)=0$. Com isso, temos:

$$
\mathcal{N}=i \mathcal{T} \operatorname{rln}\left(i \not \partial-m-\not b \gamma^{5}\right)
$$

Assim, a ação efetiva na aproximação de um laço é

$$
\begin{aligned}
& \Gamma_{e f f}=-i \mathcal{T} r \ln \left[\frac{\not D-m-\not b \gamma^{5}}{i \not \partial-m-\not b \gamma^{5}}\right]=-i \mathcal{T} r\left\{\ln \left[\frac{\not D-m-\not \partial \gamma^{5}}{i \not \partial-m-\not b \gamma^{5}}\right]+\ln \left[\frac{i \not \partial+m+\not b \gamma^{5}}{i \not \partial+m+\not b \gamma^{5}}\right]\right\} \\
& =-i \mathcal{T} r\left\{\ln \left[\left(\not D-m-\not b \gamma^{5}\right)\left(i \not \partial+m+\not b \gamma^{5}\right)\right]-\ln \left[\left(i \not \partial-m-\not b \gamma^{5}\right)\left(i \not \partial+m+\not b \gamma^{5}\right)\right]\right\}
\end{aligned}
$$

O segundo termo será desprezado, pois ele não dá contribuições à indução de Chern-Simons. Com isso, temos:

$\Gamma_{e f f}^{\prime}=-i \mathcal{T} r \ln \left[-\square-i e \not A \not \partial-m e \not A-(e \not A+2 m) \not \gamma \gamma^{5}+2 i(b . \partial) \gamma^{5}-b^{2}-m^{2}\right]$ 
e como queremos apenas termos lineares em $b_{\mu}$, usaremos a relação

$\ln (A+B) \approx \ln (A)+A^{-1} B$. Aplicando em (4.5), temos:

$\Gamma_{b}=-i \mathcal{T} r\left\{\left(\square+i e \not A \not \partial+m e \not A+m^{2}\right)^{-1}\left[(e \not A+2 m) \not b \gamma^{5}-2 i(b . \partial) \gamma^{5}\right]\right\}$.

Para calcularmos a expressão acima, usaremos o método do tempo próprio, já discutido no capítulo 3. Assim:

$\left(\square+i e \not A \not D+m e \not A+m^{2}\right)^{-1}=\int_{0}^{\infty} d \operatorname{sexp}\left[-s\left(\square+i e \not A \not D+m e \not A+m^{2}\right)\right]$,

e desta forma, reescrevemos (4.6):

$$
\Gamma_{b}=-i \mathcal{T} r \int_{0}^{\infty} d s e^{-s m^{2}} \exp \{-s(\square+i e \not A \not \partial+m e \not A)\}\left[(e \not A+2 m) \not b \gamma^{5}-2 i(b . \partial) \gamma^{5}\right]
$$

Aplicaremos agora na segunda exponencial, a relação de Baker-CampbellHausdorf em primeira ordem ${ }^{2}$,

$$
\begin{aligned}
& \exp \{-s(\square+i e \not A \not D+\text { me } \not A)\}= \\
& e^{-s \square} \cdot \exp \{-s(\text { ie } \not A \not D+m e \not A)\} \cdot \exp \left[-\frac{s^{2}}{2}[\square, \text { ie } \not A \not \partial+\text { me } \not A]\right]
\end{aligned}
$$

\footnotetext{
${ }^{2}$ há um termo não nulo de segunda ordem que é do tipo Chern-Simons mas se anula devido à antissimetria do símbolo de Levy-Civita, conforme é mostrado no apêndice D
} 
e desprezaremos termos que contenham derivada segunda do campo $A_{\mu}$. O resultado é:

$$
\begin{aligned}
& \Gamma_{b}=-i \mathcal{T} r \int_{0}^{\infty} d s e^{-s m^{2}}\left[1-s(i e \not A \not \partial+m e \not A)+\frac{s^{2}}{2}(i e \not A \not \partial+m e \not A)^{2}-\right. \\
& \left.\frac{s^{3}}{6}(i e \not A \not \partial+m e \not A)^{3}\right] \cdot\left[1-s^{2} i e\left(\partial_{\alpha} \not A\right) \not \partial \partial^{\alpha}-s^{2} m e\left(\partial_{\alpha} \not A\right) \partial^{\alpha}\right] . \\
& {\left[(e \not A+2 m) \not b \gamma^{5}-2 i(b, \partial) \gamma^{5}\right] e^{-s \square}+\ldots,}
\end{aligned}
$$

onde usamos a propriedade cíclica do traço $\mathcal{T} r(A B)=\mathcal{T} r(B A)$ e lembrando que as derivadas agem à direita. Será feito agora o desenvolvimento das potências acima afim de encontrarmos os termos de Chern-Simons, lembrando que devido à expressão acima conter um traço sobre as matrizes de Dirac, termos que contenham um número ímpar das matrizes, será nulo.

\section{Cálculo das potências de (4.10)}

Faremos aqui o cálculo explícito das potências da Eq. (4.10) até terceira ordem em A, pois termos de ordem superior não dão contribuições ao termo de Chern-Simons. Assim, temos:

$$
\begin{aligned}
& {\left[1-s(\text { ie } \not A \not \partial+m e \not A)+\frac{s^{2}}{2}(\text { ie } \not A \not \partial+m e \not A)^{2}-\frac{s^{3}}{6}(\text { ie } \not A \not \partial+m e \not A)^{3}\right] .} \\
& {\left[1-s^{2} i e\left(\partial_{\alpha} \not A\right) \not \partial \partial^{\alpha}-s^{2} m e\left(\partial_{\alpha} \not A\right) \partial^{\alpha}\right] \cdot\left[(e \not A+2 m) \not b \gamma^{5}-2 i(b, \partial) \gamma^{5}\right]} \\
& =\left[1-s(i e \not A \not D+m e \not A)+\frac{s^{2}}{2}\left(-e^{2} \not A \not D \not A \not D+i e^{2} m(\not A \not D \not A+\not A \not A \not D)+m^{2} e^{2} \not A \not A\right)\right. \\
& -\frac{s^{3}}{6}\left(-i e^{3} \not A \not \supset \not A \not D \not A \not D-m e^{3} \not A \not D \not A \not D \not A-m e^{3} \not A \not D \not A \not A \not D+i m^{2} e^{3} \not A \not D \not A \not A\right. \\
& \left.\left.-m e^{3} \not A \not A \not D \not A D+i m^{2} e^{3} \not A \not A \not D \not A+i m^{2} e^{3} \not A \not A \not A \not D+m^{3} e^{3} \not A \not A \not A\right)\right] . \\
& {\left[1-s^{2} i e\left(\partial_{\alpha} \not A\right) \not \partial \partial^{\alpha}-s^{2} m e\left(\partial_{\alpha} \not A\right) \partial^{\alpha}\right] .\left[(e \not A+2 m) \not b \gamma^{5}-2 i(b, \partial) \gamma^{5}\right] .}
\end{aligned}
$$


Separemos agora a expressão acima em duas partes, a saber, as que contém termos do tipo $A \partial A$ e outra que contém termos do tipo $A A A$

\section{-Termos bilineares em A (parte abeliana do termo de Chern-}

\section{Simons)}

Os termos referentes à parte abeliana de Chern-Simons são:

$$
\begin{aligned}
& -i s^{2} e^{2}\left(\partial_{\alpha} \not A\right) \gamma^{\nu} \not A \not \gamma \gamma^{5} \partial_{\nu} \partial^{\alpha}-i s g^{2} \not A(\not \partial \not) \not \gamma \gamma^{5}+2 i e^{2} s^{3} \not A \gamma^{\nu}\left(\partial_{\alpha} \not A\right) \not \partial(b, \partial) \gamma^{5} \partial_{\nu} \partial^{\alpha} \\
& +2 i e^{2} m^{2} s^{3} \not A \gamma^{\nu}\left(\partial_{\alpha} \not A\right) \not \partial \gamma^{5} \partial_{\nu} \partial^{\alpha}+2 i e^{2} m^{2} s^{3} \not A\left(\partial_{\alpha} \not A\right) \not \partial \not \gamma \gamma^{5} \partial^{\alpha}+ \\
& i e^{2} s^{2} \not A(\not \partial \not A) \not \partial(b, \partial) \gamma^{5}+i e^{2} m^{2} s^{2} \not A(\not \partial \not A) \not b \gamma^{5}
\end{aligned}
$$

e agora calcularemos explicitamente o primeiro termo de (4.12):

$$
\begin{aligned}
& =-\mathcal{T} r \int_{0}^{\infty} d s e^{-s m^{2}} s^{2} e^{2}\left(\gamma^{\mu} \gamma^{\nu} \gamma^{\rho} \gamma^{\sigma} \gamma^{5}\right)\left(\partial_{\alpha} A_{\mu}\right) A_{\rho} b_{\sigma} \partial_{\nu} e^{-s \square} \\
& -\left.\operatorname{trtr}_{D} \int d^{4} x\left\langle x\left|\int_{0}^{\infty} d s e^{-s m^{2}} s^{2} e^{2} t r_{D}\left(\gamma^{\mu} \gamma^{\nu} \gamma^{\rho} \gamma^{\sigma} \gamma^{5}\right)\left(\partial_{\alpha} A_{\mu}\right) A_{\rho} b_{\sigma} \partial_{\nu} \partial^{\alpha} e^{-s \square}\right| x^{\prime}\right\rangle\right|_{x=x^{\prime}} \\
& =-\operatorname{trtr}_{D} \int d^{4} x\langle x| \int(d k) \int_{0}^{\infty} d s e^{-s m^{2}} e^{s k^{2}} e^{2} s^{2} \operatorname{tr}_{D}\left(\gamma^{\mu} \gamma^{\nu} \gamma^{\rho} \gamma^{\sigma} \gamma^{5}\right) \\
& \left.\left(\partial_{\alpha} A_{\mu}\right) A_{\rho} b_{\sigma} \partial_{\nu} \partial^{\alpha}|k\rangle\left\langle k \mid x^{\prime}\right\rangle\right|_{x=x^{\prime}} \\
& =\left.4 i \epsilon^{\mu \nu \rho \sigma} e^{2} \operatorname{tr} \int d^{4} x \int(d k) \int_{0}^{\infty} d s e^{-s m^{2}+s k^{2}} s^{2}\left(\partial_{\alpha} A_{\mu}\right) A_{r h o} b_{\sigma}\left(i k_{\nu}\right)\left(i k^{\alpha}\right)\langle x \mid k\rangle\left\langle k \mid x^{\prime}\right\rangle\right|_{x=x^{\prime}} \\
& =4 i \epsilon^{\mu \nu \rho \sigma} e^{2} \operatorname{tr} \int d^{4} x \int_{0}^{\infty} d s e^{-s m^{2}} \int(d k) e^{s k^{2}}\left(-k_{\nu} k^{\alpha}\right) s^{2}\left(\partial_{\alpha} A_{\mu}\right) A_{\rho} b_{\sigma} \\
& =4 i \epsilon^{\mu \nu \rho \sigma} e^{2} \operatorname{tr} \int d^{4} x \int_{0}^{\infty} d s e^{-s m^{2}}\left(\partial_{\alpha} A_{\mu}\right) \frac{i}{32 \pi^{2} s^{3}} \delta_{\alpha}^{\nu} A_{\rho} b_{\sigma} s^{2} \\
& =4 i \epsilon^{\mu \nu \rho \sigma} e^{2} t r \int d^{4} x \int_{0}^{\infty} d s e^{-s m^{2}}\left(\partial_{\nu} A_{\mu}\right) A_{\rho} b_{\sigma} \frac{i}{32 \pi^{2} s} .
\end{aligned}
$$

$\mathrm{Na}$ expressao acima, calculamos o traço sobre o espaço coordenado e introduzimos um conjunto completo no espaço dos momentos para podermos 
trabalhar com as derivadas. De forma análoga, calculamos os outros termos, chegando à expressão do termo bilinear da ação efetiva:

$$
\begin{aligned}
& \Gamma_{2}=\Gamma_{2}^{d i v}+\Gamma_{2}^{f i n}= \\
& 4 i \epsilon^{\mu \nu \rho \sigma} e^{2} \operatorname{tr} \int d^{4} x \int_{0}^{\infty} d s e^{-s m^{2}}\left\{\left(\partial_{\nu} A_{\mu}\right) A_{\rho} b_{\sigma} \frac{i}{32 \pi^{2} s}-\left(\partial_{\nu} A_{\mu}\right) A_{\rho} b_{\sigma} \frac{i}{16 \pi^{2} s}\right. \\
& +\left(\partial_{\nu} A_{\mu}\right) A_{\rho} b_{\sigma} \frac{i}{32 \pi^{2} s}+\left(\partial_{\nu} A_{\rho}\right) A_{\mu} b_{\sigma} \frac{i}{32 \pi^{2} s}-\left(\partial_{\nu} A_{\rho}\right) A_{\mu} b_{\sigma} \frac{i}{32 \pi^{2} s}+ \\
& \left.A_{\mu}\left(\partial_{\alpha} A_{\rho}\right) b^{\alpha} \eta_{\sigma \nu} \frac{i}{8 \pi^{2} s}-A_{\mu}\left(\partial_{\nu} A_{\rho}\right) b_{\sigma} \frac{2 i m^{2}}{32 \pi^{2}}-A_{\mu}\left(\partial_{\nu} A_{\rho}\right) b_{\sigma} \frac{2 i m^{2}}{32 \pi^{2}}+A_{\mu}\left(\partial_{\nu} A_{\rho}\right) b_{\sigma} \frac{i m^{2}}{16 \pi^{2}}\right\} \\
& =\frac{e^{2}}{4 \pi^{2}} \epsilon^{\mu \nu \rho \sigma} \operatorname{Tr} \int d^{4} x A_{\mu}\left(\partial_{\nu} A_{\rho}\right) b_{\sigma} .
\end{aligned}
$$

Assim, verificamos que os termos divergentes do termo bilinear da ação efetiva se cancelam mutuamente e que o método do tempo próprio não necessita de tomarmos $m \rightarrow 0$

\section{-Termos trilineares em A (parte não abeliana do termo de Chern-}

\section{Simons)}

Escrevemos agora os termos da parte não abeliana de Chern-Simons, que são:

$$
\begin{aligned}
& -\frac{s^{2}}{2} e^{3} \not A \not D \not A \not \supset \not A \not \gamma \gamma^{5}+\frac{s^{2}}{2} m^{2} e^{3} \not A \not A \not A \not \gamma^{5}+\frac{s^{3}}{3} e^{3} \not A \not D \not A \not D \not A \not D(b, \partial) \gamma^{5}
\end{aligned}
$$

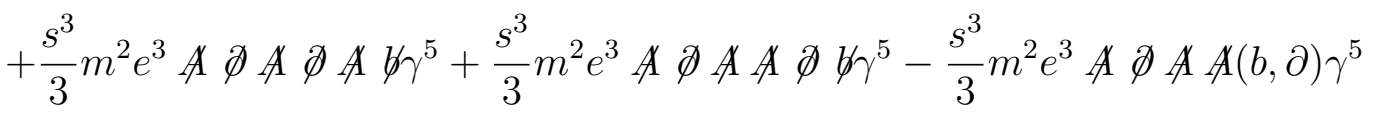

$$
\begin{aligned}
& +\frac{s^{3}}{3} m^{2} e^{3} \not A \not A \not D \not A \not D \not \gamma \gamma^{5}-\frac{s^{3}}{3} m^{2} e^{3} \not A \not A \not D \not A(b, \partial) \gamma^{5}-\frac{s^{3}}{3} m^{2} e^{3} \not A \not A \not A \not D(b, \partial) \gamma^{5} \\
& -\frac{s^{3}}{3} m^{4} e^{3} \not A \not A \not A \not \gamma^{5} \text {. }
\end{aligned}
$$

Como feito para o termo bilinear, faremos o cálculo explícito do primeiro 
termo da expressão acima,

$$
\begin{aligned}
& \left.i \operatorname{tr}_{D} \operatorname{tr} \int_{0}^{\infty} d s e^{-m^{2} s} e^{3}\left(\gamma^{\mu} \gamma^{\nu} \gamma^{\rho} \gamma^{\sigma} \gamma^{\eta} \gamma^{\lambda} \gamma^{5}\right) \frac{s^{2}}{2} A_{\mu} \partial_{\nu} A_{\rho} \partial_{\sigma} A_{\eta} b_{\lambda} e^{-s \square} \delta^{4}\left(x-x^{\prime}\right)\right|_{x=x^{\prime}} \\
& =4 i t r \int d^{4} x \int_{0}^{\infty} d s e^{-m^{2} s} \int(d k) \frac{s^{2}}{2} e^{3}\left[i \epsilon^{\lambda \mu \rho \eta} k_{\sigma} k^{\sigma} A_{\mu} A_{\rho} A_{\eta} b_{\lambda}+\right. \\
& \left.2 i \epsilon^{\lambda \sigma \mu \eta} b_{\lambda} k_{\sigma} A_{\mu} A_{\eta} k^{\rho} A_{\rho}\right] e^{s k^{2}} \\
& =-4 \operatorname{tr} \int d^{4} x \int_{0}^{\infty} d s e^{-m^{2} s} e^{3} \int(d k)\left[\frac{s^{2}}{2} \epsilon^{\lambda \mu \rho \eta} k_{\sigma} k^{\sigma} A_{\mu} A_{\rho} A_{\eta} b_{\lambda}-\right. \\
& \left.s^{2} \epsilon^{\lambda \mu \sigma \eta} A_{\mu} A_{\rho} A_{\eta} b_{\lambda} k_{\sigma} k^{\rho}\right] e^{s k^{2}} \\
& =-4 e^{3} \operatorname{tr} \int d^{4} x \int_{0}^{\infty} d s e^{-m^{2} s}\left[-\frac{i}{16 \pi^{2} s} \epsilon^{\lambda \mu \rho \eta} A_{\mu} A_{\rho} A_{\eta} b_{\lambda}+\epsilon^{\lambda \mu \rho \eta} A_{\mu} A_{\rho} A_{\eta} b_{\lambda} \frac{i}{32 \pi^{2} s}\right] \\
& =4 e^{3} \operatorname{tr} \int d^{4} x \int_{0}^{\infty} d s e^{-m^{2} s} \frac{i}{32 \pi^{2} s} \epsilon^{\lambda \mu \rho \eta} A_{\mu} A_{\rho} A_{\eta} b_{\lambda} .
\end{aligned}
$$

De forma análoga calculamos os demais termos e chegamos à seguinte expressão:

$$
\begin{aligned}
& \Gamma_{3}=-4 g^{3} \operatorname{tr} \int d^{4} x \int_{0}^{\infty} d s e^{-m^{2} s} \epsilon^{\mu \nu \rho \sigma}\left[\frac{i}{32 \pi^{2} s}-\frac{i}{32 \pi^{2} s}-m^{2} \frac{i}{32 \pi^{2}}+m^{2} \frac{2 i}{96 \pi^{2}}\right. \\
& \left.+0-m^{2} \frac{i}{96 \pi^{2}}+m^{2} \frac{2 i}{96 \pi^{2}}-m^{2} \frac{i}{96 \pi^{2}}-m^{2} \frac{i}{96 \pi^{2}}-m^{4} \frac{i s}{48 \pi^{2}}\right] A_{\mu} A_{\nu} A_{\rho} b_{\sigma} \\
& =-\frac{i g^{3}}{6 \pi^{2}} \operatorname{tr} \int d^{4} x \epsilon^{\mu \nu \rho \sigma} A_{\mu} A_{\nu} A_{\rho} b_{\sigma}
\end{aligned}
$$

e verificamos novamente que os termos divergentes se cancelam. Por fim, combinando as contribuições abeliana e não abeliana, encontramos o valor da ação efetiva:

$$
\Gamma_{C S}=\frac{e^{2}}{4 \pi^{2}} \operatorname{tr} \int d^{4} x \epsilon^{\mu \nu \rho \sigma}\left[A_{\mu} \partial_{\nu} A_{\rho} b_{\sigma}-\frac{2 i e}{3} A_{\mu} A_{\nu} A_{\rho} b_{\sigma}\right]
$$

que é finito e portanto verificamos que a adição de um campo de fundo,que quebra a simetria de Lorentz à lagrangiana da teoria de calibre, induz termos 
do tipo Chern-Simons, o que já tinha sido obtido por outros métodos de regularização. Porém com resultado numérico diferente[18].

\subsection{Gravitação}

Estamos interessados em estudar o caso de férmions num campo gravitacional, incluindo o termo de violação da simetria de Lorentz no referencial da partícula, verificar se tal modelo é possível e se há a indução de ChernSimons como ocorre na teoria de calibre. Como já foi brevemente discutido no capítulo 2, para estudarmos o campo gravitacional, temos que levar em conta a curvatura do espaço. Conseqüentemente, a lagrangeana de interação neste caso é dada por:

$$
\mathcal{L}=\bar{\psi}\left(i \not D-b \gamma^{5}-m\right) \psi
$$

onde $\not{D}=\gamma^{\mu} \mathcal{D}_{\mu}=\gamma^{a} V_{a}^{\bullet \mu}\left(\partial_{\mu}+\frac{1}{2} \sigma^{c d} V_{c}^{\bullet \nu} V_{d \nu ; \mu}\right)$ é a derivada covariante num espaço curvo, sob a presença de um campo fermiônico, como discutida no capítulo 1 e em [17], que podemos escrever como:

$$
\not D=\gamma^{a} V_{a}^{\bullet \mu}\left(\partial_{\mu}-\frac{i}{4} \omega_{\mu c d} \sigma^{c d}\right) \doteq \gamma^{a} V_{a}^{\bullet \mu} D_{\mu}
$$

Na expressão (4.20), $V_{a}^{\bullet \mu}$ é a tetrada, ou o quadrivetor do espaço tangente, $\omega_{\mu c d}$ é a conexão de spin, que é útil escrevermos como $\omega_{\mu} \doteq \frac{1}{4} \omega_{\mu c d} \sigma^{c d} \mathrm{e}$ $\sigma^{c d}=\frac{i}{2}\left[\gamma^{c}, \gamma^{d}\right]$. Com isso, a ação efetiva na aproximação de um laço é 
definida por meio da expressão:

$$
\begin{aligned}
& e^{-i \Gamma_{e f f}} \doteq \int D \bar{\psi} D \psi \exp \left[i \int \bar{\psi}\left(\not \supset-m \not b \gamma^{5}\right) \psi \sqrt{g} d^{4} x\right. \\
& \Gamma_{e f f}=-i \mathcal{T} r\left(\ln \left(i \not \partial-m-\not \partial \gamma^{5}+\not\right) \mathcal{N}\right),
\end{aligned}
$$

em que $g \doteq \operatorname{det}\left(-g_{\mu \nu}\right)$ e $\mathcal{N}$ uma constante de normalização escolhida de modo que $\Gamma_{\text {eff }}(\omega=0)=0$. Assim, temos:

$$
\begin{aligned}
& \Gamma_{e f f}=-i \mathcal{T} r \ln \left[\frac{\left(i \not D-m-\not \partial \gamma^{5}\right)}{\left(i \not \partial-m-\not b \gamma^{5}\right)}\right] \\
& -i \mathcal{T} r\left\{\ln \left[\frac{\left(i \not D-m-\not \partial \gamma^{5}\right)}{\left(i \not \partial-m-\not b \gamma^{5}\right)}\right]+\ln \left[\frac{\left(i \not \partial+m+\not b \gamma^{5}\right)}{\left(i \not \supset+m+\not b \gamma^{5}\right)}\right]\right\} \\
& =-i \mathcal{T} r\left\{\ln \left[\left(i \not D-m-\not b \gamma^{5}\right)\left(i \not \partial+m+\not b \gamma^{5}\right)\right]-\ln \left[\left(i \not \partial-m-\not b \gamma^{5}\right)\left(i \not \partial+m+\not b \gamma^{5}\right)\right]\right\} \text {. }
\end{aligned}
$$

O último termo da expressão acima será desprezado pois ele não contribui à indução de Chern-Simons. Assim,

$$
\begin{aligned}
& \Gamma_{C S}=-i \mathcal{T} \operatorname{rln}\left[-\square+i \not \phi \partial+m \not \psi-m^{2}+(\phi-2 m) \not b \gamma^{5}+2 i(b, \partial) \gamma^{5}-b^{2}\right], \\
& \approx-i \mathcal{T} r\left\{\left(-\square+i \not \not \partial+m \phi-m^{2}\right)^{-1}\left[(\phi-2 m) \not \gamma^{5}+2 i(b, \partial) \gamma^{5}\right]\right\}, \\
& =-i \mathcal{T} r \int_{0}^{\infty} d s \exp \left[-\left(\square-i \not \not \partial-m \phi+m^{2}\right) s\right] .\left[(2 m-\not) \not b \gamma^{5}-2 i(b, \partial) \gamma^{5}\right] .
\end{aligned}
$$


Agora, como feito na seção anterior, aplicaremos a fórmula de Baker-CampbellHausdorf na exponencial da Eq. (4.23):

$$
\begin{aligned}
& \exp [-s(\square-i \phi \not \partial-m \phi)]=\exp [-s \square] . \exp [-s(-i \phi \not \partial-m \psi)] . \\
& \exp \left[-\frac{s^{2}}{2}[\square,-i \phi \not \partial-m \phi]\right] \\
& \Rightarrow \Gamma_{C S}=-i \mathcal{T} r \int_{0}^{\infty} d s e^{-s m^{2}} e^{-s \square} \exp [-s(-i \phi \not \partial-m \psi)] . \\
& \exp \left\{-s^{2}\left[-i\left(\partial_{\alpha} \phi\right) \not \partial \partial^{\alpha}-m\left(\partial_{\alpha} \phi\right) \partial^{\alpha}\right]\right\}\left[(2 m-\phi) \not \gamma^{5}-2 i(b, \partial) \gamma^{5}\right] \\
& =-i \mathcal{T} r \int_{0}^{\infty} d s e^{-s m^{2}} \exp [-s(-i \psi \not \partial-m \psi)] . \\
& \exp \left\{-s^{2}\left[-i\left(\partial_{\alpha} \phi\right) \not \partial \partial^{\alpha}-m\left(\partial_{\alpha} \phi\right) \partial^{\alpha}\right]\right\}\left[(2 m-\psi) \not b \gamma^{5}-2 i(b, \partial) \gamma^{5}\right] e^{-s \square} .
\end{aligned}
$$

Na última passagem usamos a propriedade cíclica do traço. O que faremos agora é expandir em primeira ordem termos do tipo $\partial \omega$ e em terceira ordem termos do tipo $\omega$, que são os termos que nos interessam. Com isso, temos:

$$
\begin{aligned}
& \Gamma_{C S}=-i \mathcal{T} r \int_{0}^{\infty} d s e^{-s m^{2}}\left[1-s(-i \phi \not \partial-m \psi)+\frac{s^{2}}{2}(-i \phi-m \psi)^{2}-\right. \\
& \left.-\frac{s^{3}}{6}(-i \phi-m \phi)^{3}\right] \cdot\left[1-s^{2}\left(-i\left(\partial_{\alpha} \psi\right) \not \partial \partial^{\alpha}-m\left(\partial_{\alpha} \psi\right) \partial^{\alpha}\right)\right] . \\
& {\left[(2 m-\psi) \not \partial \gamma^{5}-2 i(b, \partial) \gamma^{5}\right] e^{-s \square}}
\end{aligned}
$$

\section{Dsenvolvimento de $\Gamma_{C S}$}

O que faremos aqui é o cálculo explícito dos termos entre colchetes da 
Eq.(4.26) e pegar os termos relevantes,

$$
\begin{aligned}
& {\left[1-s(-i \psi \not \partial-m \phi)+\frac{s^{2}}{2}\left(-\psi \not \partial \phi \not \partial+i m(\phi \not \partial \psi+\psi \phi \not \partial)+m^{2} \phi \psi\right)\right.} \\
& -\frac{s^{3}}{3}(i \omega \not \partial \phi \not \partial \phi \not \partial+m \psi \not \partial \phi \not \partial \phi+m \psi \phi \not \partial \phi \not \partial- \\
& \left.\left.i m^{2} \psi \phi \not \partial \phi+m \phi \not \partial \phi \psi \not \partial-i m^{2} \phi \not \partial \phi \psi-i m^{2} \phi \psi \phi \not \partial-m^{3} \psi \phi \psi\right)\right] . \\
& {\left[1-s^{2}\left(-i\left(\partial_{\alpha} \not\right) \not \partial \partial^{\alpha}-m\left(\partial_{\alpha} \not\right) \partial^{\alpha}\right)\right] .\left[(-\not \omega+2 m) \not b \gamma^{5}-2 i(b, \partial) \gamma^{5}\right] .}
\end{aligned}
$$

\section{-Termos bilineares em $\omega$ (parte abeliana do termo de Chern-Simons)}

Os termos de (4.27) que correspondem à parte abeliana de Chern-Simons são:

$$
\begin{aligned}
& -i s^{2}\left(\partial_{\alpha} \phi\right) \gamma^{\nu} \phi \not \gamma \gamma^{5} \partial_{\nu} \partial^{\alpha}-i s \psi(\not \partial \phi) \not b \gamma^{5}+2 i s^{3} \phi \gamma^{\nu}\left(\partial_{\alpha} \psi\right) \not \partial \gamma^{5}(b, \partial) \partial_{\nu} \partial^{\alpha} \\
& +2 i m^{2} s^{3} \psi \gamma^{\nu}\left(\partial_{\alpha} \psi\right) \not \gamma \gamma^{5} \partial_{\nu} \partial^{\alpha}+2 i m^{2} s^{3} \phi\left(\partial_{\alpha} \phi\right) \gamma^{\rho} \not b \gamma^{5} \partial_{\rho} \partial^{\alpha} \\
& +i s^{2} \psi(\not \partial \psi) \gamma^{\sigma}\left(b_{\lambda} \partial^{\lambda}\right) \partial_{\sigma} \gamma^{5}+i m^{2} s^{2} \psi(\not \phi \psi) \not \gamma^{5} .
\end{aligned}
$$

Como na teoria de calibre, termos proporcionais a m são finitos ao passo que os termos que não são proporcionais a m são divergentes, os quais se cancelam mutuamente. Assim, um cálculo análogo ao feito no caso da teoria de calibre nos mostra que

$$
\Gamma_{2}=\frac{1}{32 \pi^{2}} \int d^{4} x \sqrt{g} \epsilon^{\mu \nu \rho \sigma} b_{\mu} \partial_{\nu} \omega_{\rho a c} \omega_{\sigma}^{\bullet c a}
$$


-Termos trilineares em $\omega$ (parte não abeliana do termo de Chern-

\section{Simons)}

$$
\begin{aligned}
& \frac{s^{2}}{2} \psi \gamma^{\nu} \phi \gamma^{\sigma} \phi \not \gamma \gamma^{5} \partial_{\nu} \partial_{\sigma}-\frac{s^{2}}{2} m^{2} \phi \psi \phi \not \gamma \gamma^{5}- \\
& \frac{s^{3}}{3} \phi \gamma^{\nu} \psi \gamma^{\sigma} \phi \not \partial \partial_{\nu} \partial_{\sigma} \gamma^{5}(b, \partial)-\frac{s^{3}}{3} m^{2} \psi \gamma^{\nu} \phi \gamma^{\sigma} \phi \not \gamma^{5} \partial_{\nu} \partial_{\sigma}-\frac{s^{3}}{3} m^{2} \psi \psi \gamma^{\rho} \psi \not \partial \not \gamma^{5} \partial_{\rho} \\
& +\frac{s^{3}}{3} m^{2} \psi \psi \gamma^{\rho} \psi \gamma^{5}(b, \partial) \partial_{\rho}-\frac{s^{3}}{3} m^{2} \psi \gamma^{\nu} \psi \psi \not \partial \not \gamma^{5} \partial_{\nu}+\frac{s^{3}}{3} m^{2} \psi \gamma^{\nu} \psi \psi \gamma^{5}(b, \partial) \partial_{\nu} \\
& +\frac{s^{3}}{3} m^{2} \psi \psi \phi \not \partial \gamma^{5}(b, \partial)+\frac{s^{3}}{3} m^{4} \psi \psi \psi \not \gamma^{5}
\end{aligned}
$$

Analogamente ao caso da teoria de calibre, os termos que não são proporcionais a m apresentam divergência e os termos proporcionais a m não. Com isso, temos

$$
\Gamma_{3}=\Gamma_{3}^{f i n}+\Gamma_{3}^{\text {div }}
$$

e verificamos que a parte divergente cancela-se, resultando os seguintes termos para a parte trilinear:

$$
\begin{aligned}
& \Gamma_{3}=i \operatorname{tr}_{D} \int d^{4} x \sqrt{g} \int_{0}^{\infty} d s e^{-s m^{2}}\left[\frac{s^{2}}{2} m^{2} \phi \psi \phi \not \gamma \gamma^{5}+\right. \\
& \frac{s^{3}}{3} m^{2} \psi \gamma^{\nu} \phi \gamma^{\sigma} \phi \not \gamma \gamma^{5} \partial_{\nu} \partial_{\sigma}+\frac{s^{3}}{3} m^{2} \phi \psi \gamma^{\rho} \phi \not \partial \not \gamma \gamma^{5} \partial_{\rho} \\
& +\frac{s^{3}}{3} m^{2} \psi \gamma^{\nu} \phi \psi \not \partial \not \gamma \gamma^{5} \partial_{\nu}-\frac{s^{3}}{3} m^{2} \psi \phi \gamma^{\rho} \phi \gamma^{5}(b, \partial) \partial_{\rho}-\frac{s^{3}}{3} m^{2} \phi \gamma^{\nu} \phi \psi \gamma^{5}(b, \partial) \partial_{\nu} \\
& \left.-\frac{s^{3}}{3} m^{2} \psi \psi \phi \not \partial \gamma^{5}(b, \partial)-\frac{s^{3}}{3} m^{4} \psi \psi \phi \not \gamma^{5}\right]\left.e^{-s \square} \delta\left(x-x^{\prime}\right)\right|_{x=x^{\prime}} .
\end{aligned}
$$


Assim, após o cálculo do traço, a expressão acima pode ser escrita como

$$
\begin{aligned}
& -\frac{i}{16} \int d^{4} x \sqrt{g} \int_{0}^{\infty} d s e^{-s m^{2}}\left[b _ { \mu } \omega _ { \nu a b } \omega _ { \lambda c d } \omega _ { \rho e f } \int ( d k ) e ^ { s k ^ { 2 } } \left(\frac{s^{2}}{2} m^{2} \epsilon^{\mu \nu \lambda \rho}+\frac{s^{3}}{3} m^{2} \epsilon^{\mu \nu \lambda \rho} k^{2}+\right.\right. \\
& \left.\epsilon^{\alpha \nu \lambda \rho} k_{\alpha} k^{\mu}-\frac{s^{3}}{3} m^{4} \epsilon^{\mu \nu \lambda \rho}\right) \cdot\left[g^{a f}\left(g^{b c} g^{d e}-g^{b d} g^{c e}\right)+g^{a e}\left(g^{b d} g^{c f}-g^{b c} g^{d f}\right)+\right. \\
& \left.\left.g^{a d}\left(g^{b f} g^{c e}-g^{b e} g^{c f}\right)+g^{a c}\left(g^{b e} g^{d f}-g^{b f} g^{d e}\right)\right]\right]
\end{aligned}
$$

E integrando sobre o momento $\mathrm{k}$ e sobre o parâmetro $\mathrm{s}$, obtemos a parte trilinear da ação efetiva

$$
\Gamma_{3}=-\frac{1}{48 \pi^{2}} \int d^{4} x \sqrt{g} \epsilon^{\mu \nu \lambda \rho} b_{\mu} \omega_{\nu a b} \omega_{\lambda}^{\bullet b c} \omega_{\rho c}^{\bullet \bullet} .
$$

Combinando este resultado com o termo bilinear, vemos que o termo induzido do modelo de Chern-Simons na ação efetiva da gravitação é dado por

$$
\Gamma_{C S}=\frac{1}{32 \pi^{2}} \int d^{4} x \sqrt{g} \epsilon^{\mu \nu \lambda \rho} b_{\mu}\left(\partial_{\nu} \omega_{\lambda a b} \omega_{\rho}^{\bullet b a}-\frac{2}{3} \omega_{\nu a b} \omega_{\lambda}^{\bullet b c} \omega_{\rho c}^{\bullet \bullet a}\right)
$$

Assim, verificamos que ao adicionarmos um campo de fundo que quebra a simetria de Lorentz na lagrangeana de interação de férmions com um campo gravitacional, surgem termos induzidos do tipo Chern-Simons. 


\section{Capítulo 5}

\section{Conclusão}

Aplicamos o método do tempo próprio para o cálculo da ação efetiva em $(3+1)$ D na teoria de calibre e na gravitação com a introdução de um campo de fundo que quebra a simetria de Lorentz. Verificamos com isso que correções radiativas em um laço induzem termos do tipo Chern-Simons em ambas as teorias como era esperado e que estas contribuições são finitas. Vimos também que os resultado $\Gamma_{C S}=\frac{e^{2}}{4 \pi^{2}} \operatorname{Tr} \int d^{4} x \epsilon^{\mu \nu \rho \sigma}\left[A_{\mu} \partial_{\nu} A_{\rho} b_{\sigma}-\frac{2 i g}{3} A_{\mu} A_{\nu} A_{\rho} b_{\sigma}\right]$ obtido na teoria de calibre é o mesmo obtido na referência [7], através de um método diferente de regularização. Já na gravitação, o resultado obtido, a saber, $\Gamma_{C S}=\frac{1}{32 \pi^{2}} \int d^{4} x \epsilon^{\mu \nu \lambda \rho} b_{\mu}\left(\partial_{\nu} \omega_{\lambda a b} \omega_{\rho}^{\bullet b a}-\frac{2}{3} \omega_{\nu a b} \omega_{\lambda}^{\bullet b c} \omega_{\rho c}^{\bullet \bullet a}\right)$, difere do resultado obtido anteriormente em [18], por um outro método de regularização. ${ }^{1}$ É importante ressaltar que adicionamos um campo de fundo que poderia in-

\footnotetext{
${ }^{1}$ Essa ambigüidade no coeficiente do termo de Chern-Simons induzido, por diferentes métodos de regularização já era conhecido [15] fora do contexto de quebra de Lorentz.
} 
duzir termos do tipo Chern-Simons, uma vez que o termo $\not b \gamma^{5}$ na lagrangiana, conduz a $\operatorname{tr}_{D}\left(\gamma^{\mu} \gamma^{\nu} \gamma^{\rho} \gamma^{\sigma}\right)$. Lembrando também que nosso resultado foi obtido sem impor quaisquer aproximações, como tomarmos $\lim m \rightarrow 0$, uma vez que pelo método usado, as contribuições divergentes se cancelam mutuamente. Por fim, ao verificarmos a indução de Chern-Simons, poderíamos ter campos eletromagnético e gravitacional massivos. Porém, publicações recentes mostram que, embora possível, os valores de massa seriam extremamente pequenos[9], bem como a violação da simetria de Lorentz é algo praticamente imperceptível nas nossas escalas típicas de energia. 


\section{Apêndice A}

\section{Variáveis de Grassmann}

No estudo do campo fermiônico, é importante a introdução de variáveis anticomutantes, que introduziremos brevemente a seguir. Sejam $\theta$ e $\eta$ duas variáveis de Grassmann,

$$
\theta \cdot \eta=-\eta \cdot \theta
$$

ou seja, $\theta^{2}=0$. Um produto de duas variáveis de Grassmann comuta com outra variável de Grassmann e a soma dessas variáveis ou a multiplicação de uma variável de Grassmann por um número puro segue as mesmas regras de adição e multiplicação por um escalar do cálculo vetorial. Queremos também eh integrar essas variáveis para poder trabalhar com elas no tratamento dos 
férmions e para isso, usaremos a seguintes definições:

$$
\begin{aligned}
\int d \theta & \equiv 0 \\
\int d \theta(\theta) & \equiv 1,
\end{aligned}
$$

e quando estamos trabalhando com mais de uma variável de Grassmann, temos:

$$
\int d \theta \int d \eta(\eta \theta)=1
$$

É interessante defirnirmos as variáveis de Grassmann num espaço complexo, uma vez que o campo de Dirac está no plano complexo. Assim, temos:

$$
(\theta \eta)^{*} \equiv \eta^{*} \theta^{*}=-\theta^{*} \eta^{*}
$$

e definiremos as variáveis de modo conveniente como:

$$
\theta=\frac{\theta_{1}+i \theta_{2}}{\sqrt{2}}, \theta^{*}=\frac{\theta_{1}-i \theta_{2}}{\sqrt{2}}
$$

tal que

$$
\begin{aligned}
& \int d \theta^{*} d \theta\left(\theta \theta^{*}\right)=\int d \theta_{1} d \theta_{2} J\left(\frac{\partial\left(\theta^{*}, \theta\right)}{\partial\left(\theta_{2}, \theta_{1}\right)}\right)-i \frac{\theta_{1} \theta_{2}}{2}+i \frac{\theta_{2} \theta_{1}}{2} \\
& =-i \int d \theta_{1} d \theta_{2} i \theta_{2} \theta_{1}=1 .
\end{aligned}
$$

Agora, calculemos a integral gaussiana sobre as variáveis de Grassmann:

$$
\begin{aligned}
& \int d \theta^{*} d \theta \exp \left(-\theta^{*} b \theta\right) \\
& =\int d \theta^{*} d \theta\left(1-\theta^{*} b \theta\right) \\
& =\int d \theta^{*} d \theta\left(1+\theta \theta^{*} b\right) \\
& =\int d \theta^{*} d \theta\left(\theta \theta^{*} b\right)=b,
\end{aligned}
$$


lembrando que a exponencial de Grassmann possui apenas termos lineares em $\theta \theta^{*}$, já que $\left(\theta \theta^{*}\right)^{2}=0$ e que por isso a igualdade na segunda linha da Eq. (A.8) acima é válida. E, vemos que há uma mudança com relação a variáveis ordinárias, uma vez que com estas o resultado seria com o termo constante no denominador. Contudo, verificamos que

$$
\begin{aligned}
& \int d \theta^{*} d \theta\left(\theta \theta^{*}\right) \exp \left(-\theta^{*} b \theta\right) \\
& =\int d \theta^{*} d \theta\left(\theta \theta^{*}\right)\left(1-b \theta^{*} \theta\right) \\
& =\int d \theta^{*} d \theta\left(\theta \theta^{*} b\right)\left(\frac{1}{b}+\theta \theta^{*}\right)=b \cdot \frac{1}{b}=1
\end{aligned}
$$

o fator $\theta \theta^{*}$ introduz um fator $\frac{1}{b}$ como nas integrais Gaussianas ordinárias. Agora, desenvolveremos a integral Gaussiana para n variáveis de Grassmann e começaremos considerando n variáveis de Grassmann $\theta_{i}$ e uma matriz unitária tal que $\eta_{i}=U_{i j} \theta_{j}$. Assim,

$$
\begin{aligned}
& \prod_{i=1}^{n} \eta_{i}=\frac{1}{n !} \epsilon^{i j \ldots l} \eta_{1} \eta_{2} \ldots \eta_{l} \\
& =\frac{1}{n !} \epsilon^{i j \ldots l} U_{i i^{\prime}} \theta_{i^{\prime}} U_{j j^{\prime}} \theta_{j^{\prime}} \ldots U_{l l^{\prime}} \theta_{l^{\prime}} \\
& =\frac{1}{n !} \epsilon^{i j \ldots l} U_{i i^{\prime}} U_{j j^{\prime}} \ldots U_{l l^{\prime}} \epsilon^{i^{\prime} j^{\prime} \ldots l^{\prime}}\left(\prod_{i=1}^{n} \theta_{i}\right) \\
& =(\operatorname{det} U)\left(\prod_{i=1}^{n} \theta_{i}\right) .
\end{aligned}
$$

Numa integral geral

$$
\int D \theta^{*} D \theta\left(f\left(\theta^{*}, \theta\right)\right)
$$


o único termo que restará, será aquele proporcional a $\prod_{i} \theta_{i} \prod_{i} \theta_{i}^{*}$ pois os outros anular-se-ão devido à propriedade das variáveis de Grassmann. Assim, consideremos a integral Gaussiana:

$$
\int\left(D \theta^{*} D \theta\right) \exp \left(-\theta_{i}^{*} b_{i j} \theta_{j}\right)
$$

na qual, diagonalizaremos a matriz B, chamando-a de B'. Com isso, nossa integral fica:

$$
\begin{aligned}
& \int\left(D \theta^{*} D \theta\right) \exp \left(-\theta_{i}^{*} b_{i i}^{\prime} \theta_{i}\right) \\
& =\int\left(D \theta^{*} D \theta\right) \exp \left(-\sum_{i} \theta^{*} b_{i i}^{\prime} \theta_{i}\right) \\
& =\prod_{i} b_{i i}^{\prime}=\operatorname{det} B^{\prime}=\operatorname{det} B .
\end{aligned}
$$

Verificamos que, como no caso de duas variáveis, o determinante aparece no numerador, ao passo que no caso de variáveis ordinárias, o determinante aparece no denominador. 


\title{
Apêndice B
}

\section{Prova da identidade}

\author{
$\ln \operatorname{det} B=\operatorname{trln} B$
}

Seja

$$
\mathbf{C}_{\mathbf{d}}=\left[\begin{array}{ccc}
c_{1} & \ldots & 0 \\
\vdots & \ddots & \vdots \\
0 & \ldots & c_{n}
\end{array}\right]
$$

diagonal e $B_{d}$ definido como:

$$
B_{d}=I+C_{d}+\frac{1}{2} C_{d} C_{d}+\ldots
$$


ou seja, $B_{d} \equiv e^{C_{d}}$, o que, por abuso de notação, podemos escrever $C_{d}=\ln B_{d}$. Como $C_{d} \ldots C_{d}$ multiplicado m vezes é igual a

$$
\mathbf{C}_{\mathbf{d}}=\left[\begin{array}{ccc}
c_{1}^{m} & \ldots & 0 \\
\vdots & \ddots & \vdots \\
0 & \ldots & c_{n}^{m}
\end{array}\right]
$$

temos

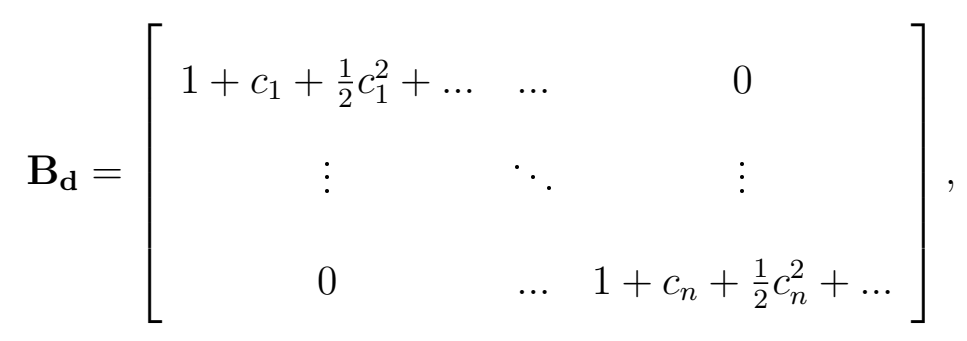

o que gera uma matriz diagonal, cujos termos sao

$$
\mathbf{B}_{\mathbf{d}}=\left[\begin{array}{ccc}
e^{c_{1}} & \ldots & \cdots \\
\vdots & \ddots & \vdots \\
0 & \ldots & e^{c_{n}}
\end{array}\right]
$$

Ou seja, a matriz $B_{d}$ é diagonal e tal que $b_{i}=e^{c_{i}}$. Multipliquemos agora a Eq.(B.1) à esquerda por uma matriz unitária inversível $U$ e à direita por $U^{-1}$. Assim,

$$
\begin{aligned}
& U B_{d} U^{-1}=U I U^{-1}+U C_{d} U^{-1}+\frac{1}{2} U C_{d} C_{d} U^{-1}+\ldots \\
& =I+U C_{d} U^{-1}+\frac{1}{2} U C_{d} U U^{-1} C_{d} U^{-1}+\ldots
\end{aligned}
$$


e sejam $C \equiv U C_{d} U^{-1}$ e $B \equiv U B_{d} U^{-1}$ as matrizes não diagonais resultantes.

Ou seja, $B=e^{C}$, que podemos escrever como $C \equiv \ln (B)^{1}$. Com isso, temos:

$$
\begin{aligned}
& \ln (\operatorname{det}(B))=\ln \left(\operatorname{det}\left(U B_{d} U^{-1}\right)\right)=\ln \left(\operatorname{det}(U) \operatorname{det}\left(C_{d}\right) \operatorname{det}\left(U^{-1}\right)\right)=\ln \left(\operatorname{det}\left(B_{d}\right)\right) \\
& \ln \left(\prod_{i=1}^{n} b_{i}\right)=\ln \left(\exp \left(\sum_{i=1}^{n} c_{i}\right)\right)=\sum_{i=1}^{n} c_{i} \\
& =\operatorname{tr}\left(C_{d}\right) .
\end{aligned}
$$

Mas se $C=U C_{d} U^{-1}$, então $C_{d}=U^{-1} C U$ e portanto

$$
\ln (\operatorname{det}(B))=\operatorname{tr}\left(U^{-1} C U\right)=U_{i j}^{-1} C_{j k} U_{k i}
$$

e, como são elementos de matrizes, posso por na ordem que me for mais conveniente, assim:

$$
\begin{aligned}
& \ln (\operatorname{det}(B))=C_{j k} U_{k i} U_{i j}^{-1}=C_{j k} \delta_{k j} \\
& =C_{i i} .
\end{aligned}
$$

Lembrando que índices repetidos indicam somatória sobre esses índices e combinando com a definição da matriz C, temos:

$$
C_{i i}=\operatorname{tr}(C)=\operatorname{tr}(\ln (B))
$$

como queríamos demonstrar.

\footnotetext{
${ }^{1}$ Lembrando que B e C são matrizes, sendo portanto uma notação e não a exponencial e o logaritmo como convencionalmente os conhecemos.
} 
72 APENDICE B. PROVA DA IDENTIDADE LN DET B = TRLNB 


\section{Apêndice C}

\section{Obtenção de $\operatorname{Tr} \mathcal{O}\left(\mathrm{x}, \partial_{X}\right)=$}

$$
\int \frac{d^{D} x}{(2 \pi)^{D}} \mathcal{O}\left(\partial_{x}+i k, x\right)
$$

Consideremos como exemplo um operador $\mathcal{O}$ definido por:

$$
\mathcal{O}\left(x, \partial_{x}\right) \doteq\left(\partial_{x}+f \partial_{x}+g\right)^{2}
$$

sendo $\mathrm{f}(\mathrm{x})$ e $\mathrm{g}(\mathrm{x})$ funções quaisquer de $\mathrm{x}$ e $\partial_{x} \equiv \frac{d}{d x}$ o operador de derivação na variável x. Como a derivada atua em tudo à sua direita, temos:

$$
\begin{aligned}
& \partial_{x} f=\left(\partial_{x} f\right)+f \partial_{x} \\
& \partial_{x}^{2} f=\partial_{x}\left(\left(\partial_{x} f\right)+f \partial_{x}\right) \\
& =\left(\partial_{x}^{2} f\right)+2\left(\partial_{x} f\right) \partial_{x}+f \partial_{x}^{2} .
\end{aligned}
$$


74 APÊNDICE C. OBTENÇÃO DE TRO $\left(X, \partial_{X}\right)=\int \frac{D^{D} X}{(2 \pi)^{D}} \mathcal{O}\left(\partial_{X}+I K, X\right)$ Assim, expandindo (C.1):

$$
\begin{aligned}
& \mathcal{O}=\partial_{x}^{4}+\left(\partial_{x}^{2} f\right) \partial_{x}+2\left(\partial_{x} f\right) \partial_{x}^{2}+ \\
& +f \partial_{x}^{3}+\left(\partial_{x}^{2} g\right)+2\left(\partial_{x} g\right) \partial_{x}+g \partial_{x}^{2}+ \\
& +f \partial_{x}^{3}+f\left(\partial_{x} f\right) \partial_{x}+f f \partial_{x}^{2}+ \\
& +f\left(\partial_{x} g\right)+f g \partial_{x}+g \partial_{x}^{2}+g f \partial_{x}+g g .
\end{aligned}
$$

O traço do operador $\mathcal{O}$ pode ser escrito na representação das posições,

$$
\begin{aligned}
& \operatorname{Tr} \mathcal{O} \doteq \int d^{3} x\langle x|\mathcal{O}| x\rangle \\
& =\int d^{3} x(d k)\left[\langle x \mid k\rangle\left\langle k\left|\partial_{x}^{4}\right| x\right\rangle+\left\langle x\left|\left(\partial_{x}^{2} f\right)\right| k\right\rangle\left\langle k\left|\partial_{x}\right| x\right\rangle+\right. \\
& +2\left\langle x\left|\left(\partial_{x} f\right)\right| k\right\rangle\left\langle k\left|\partial_{x}^{2}\right| x\right\rangle+\langle x|f| k\rangle\left\langle k\left|\partial_{x}^{3}\right| x\right\rangle+ \\
& +\left\langle x\left|\left(\partial_{x}^{2} g\right)\right| k\right\rangle\langle k \mid x\rangle+2\left\langle x\left|\left(\partial_{x} g\right)\right| k\right\rangle\left\langle k\left|\partial_{x}\right| x\right\rangle+ \\
& +\langle x|g| k\rangle\left\langle k\left|\partial_{x}^{2}\right| x\right\rangle+\langle x|f| k\rangle\left\langle k\left|\partial_{x}^{3}\right| x\right\rangle+ \\
& +\left\langle x\left|f\left(\partial_{x} f\right)\right| k\right\rangle\left\langle k\left|\partial_{x}\right| x\right\rangle+\left\langle x\left|f^{2}\right| k\right\rangle\left\langle k\left|\partial_{x}^{2}\right| x\right\rangle+ \\
& +\left\langle x\left|f\left(\partial_{x} g\right)\right| k\right\rangle\langle k \mid x\rangle+\langle x|f g| k\rangle\left\langle k\left|\partial_{x}\right| x\right\rangle+ \\
& +\langle x|g| k\rangle\left\langle k\left|\partial_{x}^{2}\right| x\right\rangle+\langle x|g f| k\rangle\left\langle k\left|\partial_{x}\right| x\right\rangle+ \\
& +\langle x|g g| k\rangle\langle k \mid x\rangle]
\end{aligned}
$$

onde introduzimos conjuntos completos $1=\int(d k)|k\rangle\langle k|$. Usando a representação de ondas planas para as expressões acima:

$$
\langle x \mid k\rangle=e^{-i k \cdot x}=\langle k \mid x\rangle^{*}
$$


e lembrando que o produto escalar é um produto de Minkowski,

$$
k . x=k^{0} x^{0}-k_{i} x^{i},
$$

temos,

$$
\left\langle k\left|\partial_{\mu}\right| x\right\rangle=\partial_{\mu}\langle k \mid x\rangle=i k_{\mu}\langle k \mid x\rangle .
$$

Aplicando agora (C.7) em (C.4), obtemos:

$$
\begin{aligned}
& \operatorname{Tr} \mathcal{O}=\int d^{3} x(d k)\langle x \mid k\rangle\langle k \mid x\rangle \\
& \cdot\left[(i k)^{4}+\partial_{x}^{2} f(i k)+2 \partial_{x} f(i k)^{2}+f(i k)^{3}\right. \\
& +\partial_{x}^{2} g+2 \partial_{x} g(i k)+g(i k)^{2}+f(i k)^{3} \\
& +f \partial_{x} f(i k)+f^{2}(i k)^{2}+f \partial_{x} g+f g(i k)+g(i k)^{2} \\
& \left.+g f(i k)+g^{2}\right] .
\end{aligned}
$$

Por outro lado, temos que:

$$
\begin{aligned}
& \mathcal{O}\left(\partial_{x}+i k, x\right) \cdot 1=\left(\left(\partial_{x}+i k\right)^{2}+f\left(\partial_{x}+i k\right)+g\right) . \\
& .\left(\left(\partial_{x}+i k\right)^{2}+f\left(\partial_{x}+i k\right)+g\right) \cdot 1 \\
& =\left(\partial_{x}^{2}+2 i k \partial_{x}+(i k)^{2}+f \partial_{x}+f(i k)+g\right) . \\
& .\left((i k)^{2}+f(i k)+g\right) .1
\end{aligned}
$$


76 APENDICE C. OBTENÇÃO DE TRO $\left(X, \partial_{X}\right)=\int \frac{D^{D} X}{(2 \pi)^{D}} \mathcal{O}\left(\partial_{X}+I K, X\right)$ onde no último termo entre parênteses foram omitidos os termos que, aplicados ao operador 1 dão resultados nulos. Assim temos:

$$
\begin{aligned}
& =\partial_{x}^{2} f \cdot i k+\partial_{x}^{2}+2 \partial_{x} f(i k)^{2}+2 \partial_{x} g \cdot i k+f(i k)^{3} \\
& +g(i k)^{2}+f \partial_{x} f \cdot i k+f \partial_{x} g+f(i k)^{3}+f^{2}(i k)^{2} \\
& +f g . i k+g f . i k+g^{2}+(i k)^{4}+g(i k)^{2}
\end{aligned}
$$

que é exatamente igual à Eq.(C.8). Portanto,

$$
\begin{aligned}
& \operatorname{Tr} \mathcal{O}\left(\partial_{x}, x\right) \equiv \int d^{3} x\langle x|\mathcal{O}(\partial, x)| x\rangle \\
& =\int d^{3} x(d k) \mathcal{O}\left(\partial_{x}+i k, x\right) .1 .
\end{aligned}
$$

e este resultado pode ser extendido para um espaço de dimensão D qualquer, desde que a relação de completeza seja satisfeita. 


\section{Apêndice D}

\section{Aproximação da série de}

\section{Baker-Campbell-Hausdorff}

A série de Baker-Campbell-Hausdorff é uma série infinita de comutadores definida por:

$$
\begin{aligned}
& e^{-s(X+Y)}=e^{-s X} \cdot e^{-s Y} \cdot e^{-\frac{-s^{2}}{2}[X, Y]} \cdot e^{-\frac{s^{3}}{3 !}[2 Y+X,[X, Y]]} \\
& e^{-\frac{s^{4}}{4 !}([[[X, Y], X], X+3 Y]+3[[[X, Y], Y], Y])} \ldots
\end{aligned}
$$

Em particular nesta dissertação temos:

$$
\begin{aligned}
& X \doteq \square \\
& Y \doteq i e \not A \not \partial+m e \not A,
\end{aligned}
$$

e apenas o termo de primeira ordem no comutador (Eq.(4.9)) e o termo $\left.[Y,[X, Y]] \approx 2\left(Y\left(\partial^{\alpha} Y\right)-\left(\partial^{\alpha} Y\right) Y\right)\right) \partial_{\alpha}=2 Z^{\alpha} \partial_{\alpha}$, em que $Z^{\alpha} \doteq Y \partial^{\alpha} Y-$ 
$\partial^{\alpha} Y Y$, são não nulos e contribuem com termos do tipo Chern-Simons. A contribuição do fator acima na ação efetiva é:

$$
\Gamma_{z}=-\left.i \operatorname{Tr} \int d s e^{-s m^{2}} \int d^{3} x\left(-\frac{2}{3}\right) s^{3} Z^{\alpha}(2 m \not b-2 i b . \partial) \gamma^{5} \partial_{\alpha} \delta\left(x-x^{\prime}\right)\right|_{x=x} \text { (.D.3) }
$$

Dividiremos o integrando de (D.3) em duas partes, a saber,

$$
\begin{aligned}
& (1)=Z^{\alpha} 2 m \not b \gamma^{5} \partial_{\alpha}=2 m^{2} i\left(\not \not \not \partial \partial^{\alpha} \not A \not \gamma \gamma^{5}+\right. \\
& +\not A\left(\partial^{\alpha} \not A\right) \not \partial \not \gamma \gamma^{5}-\left(\partial^{\alpha} \not A\right) \not \partial \not A \not \gamma \gamma^{5} \\
& \left.-\left(\partial^{\alpha} \not A\right) \not A \not \supset \not \gamma \gamma^{5}\right) \partial_{\alpha}
\end{aligned}
$$

e

$$
\begin{aligned}
& (2)=Z^{\alpha}(-2 i b . \partial) \gamma^{5} \partial^{\alpha}=2 i\left(\not A \not \partial\left(\partial^{\alpha} \not A\right) \not \partial \gamma^{5}\right. \\
& \left.-\left(\partial^{\alpha} \not A\right) \not \partial \not A \gamma^{5}\right) b . \partial \partial_{\alpha} .
\end{aligned}
$$

Como estes termos estão sujeitos ao traço sobre as matrizes de Dirac, temos que

$$
\begin{aligned}
(1)=\quad & 2 m^{2} i\left(\gamma^{\mu} \gamma^{\nu} \gamma^{\sigma} \gamma^{\rho} \gamma^{5}\right)\left(A_{\mu} \partial^{\alpha} A_{\sigma} b_{\rho} \partial_{\nu}+A_{\mu} \partial^{\alpha} A_{\nu} b_{\rho} \partial_{\sigma}\right. \\
& \left.-\partial^{\alpha} A_{\mu} A_{\sigma} b_{r h o} \partial_{\nu}-\partial^{\alpha} A_{\mu} A_{\nu} b_{\rho} \partial_{\sigma}\right)
\end{aligned}
$$

e vemos facilmente que o segundo fator da expressao acima é simétrico dois a dois (simétricos na troca de $\nu$ por $\sigma$ ) ao passo que o traço sobre as matrizes de Dirac apresenta um símbolo de Levi Civita, que é antissimétrico na mesma 
troca, o que anula este termo na ação efetiva. Analogamente para (2) temos:

$$
\begin{aligned}
& 2 i\left(\gamma^{\mu} \gamma^{\nu} \gamma^{\sigma} \gamma^{\rho} \gamma^{5}\right) \partial_{\alpha}\left(A_{\mu} \partial^{\alpha} A_{\sigma} \partial_{\nu} \partial_{\rho}-\right. \\
& \left.-\partial^{\alpha} A_{\mu} A_{\sigma} \partial_{\nu} \partial_{\rho}\right) b . \partial
\end{aligned}
$$

onde há uma simetria na troca de $\nu$ por $\rho$, o que cancela este termo devido à antissimetria do traço das matrizes de Dirac. 
80APÊNDICE D. APROXIMAÇÃO DA SÉRIE DE BAKER-CAMPBELL-HAUSDORFF 


\section{Apêndice E}

\section{Integrais D-dimensionais}

Faremos neste apêndice o cálculo explícito das integrais usadas no cap.4.

Consideremos inicialmente a seguinte integral:

$$
\int \frac{d^{D} k}{(2 \pi)^{D}} e^{s k^{2}}
$$

O elemento de volume em D dimensões euclideanas é dado por:

$$
d^{D} k=k^{D-1} \operatorname{sen}^{D-2}\left(\theta_{D-2}\right) \ldots \operatorname{sen}^{2}\left(\theta_{2}\right) \operatorname{sen}\left(\theta_{1}\right) \prod_{i=1}^{D-2} d \theta_{i} d \phi d k
$$

com $\mathrm{k}$ variando de 0 a $\infty, \theta$ variando de 0 a $\pi$ e $\phi$ variando de 0 a $2 \pi$, cobrindo toda a hiperesfera no espaço dos momentos. Como nossas integrais são simétricas nos ângulos, as integrais em $\theta$ e $\phi$, são iguais a $\frac{2 \pi^{D / 2}}{\Gamma(D / 2)}$ e como 
no nosso caso, temos a integral originalmente em $(3+1) \mathrm{D}$ :

$$
\begin{aligned}
& \int \frac{d^{4} k}{\left(2 \pi^{4}\right)} e^{s k^{2}}=i \int \frac{d^{4} k}{\left(2 \pi^{4}\right)} e^{-s k^{2}} \\
& =\frac{i}{(2 \pi)^{4}} \int d \theta_{1} d \theta_{2} d \phi d k\left\|k^{3}\right\| \operatorname{sen}^{2}\left(\theta_{2}\right) \operatorname{sen}\left(\theta_{1}\right) e^{-s k^{2}} \\
& =\frac{i}{8 \pi^{2}} \int k^{3} e^{-s k^{2}} d k,
\end{aligned}
$$

onde na primeira igualdade, aplicamos uma rotação de Wick, levando o quadrivetor $k=\left(k^{0},-\vec{k}\right)$ ao espaço euclideano, no qual $k^{0}=i k^{4}$, mantendo inalterada a parte espacial e introduzindo um sinal negativo na exponencial, pois $\left.\left.k^{2}=\left(k^{0}\right)^{2}-(\vec{k})^{2}\right)=\left(i k^{4}\right)^{2}-(\vec{k})^{2}\right)=-k^{2}$. Com isso, temos:

$$
\begin{aligned}
& \frac{i}{8 \pi^{2}} \int k^{3} e^{-s k^{2}} d k=\frac{i}{16 \pi^{2}} \int k^{2} e^{-s k^{2}} d k^{2} \\
& =-\frac{i}{16 \pi^{2}} \int \frac{d}{d s} e^{-s k^{2}} d k^{2}=-\frac{i}{16 \pi^{2}} \frac{d}{d s} \int e^{-s k^{2}} d k^{2} \\
& =-\frac{i}{16 \pi^{2}} \frac{d}{d s}\left(\frac{1}{s}\right)=\frac{i}{16 \pi^{2} s^{2}} .
\end{aligned}
$$

Para a integral $\int \frac{d^{4} k}{(2 \pi)^{4}} k_{\mu} k_{\nu} e^{s k^{2}}$ o processo é análogo, exceto que temos que adicionar um tensor simétrico ao resultado, uma vez que o tensor $k_{\mu} k_{\nu}$ desaparece ao calcularmos a integral e o único tensor simétrico de segunda ordem disponível é o tensor de Minkowski. Então usamos o seguinte anzats:

$$
\int \frac{d^{4} k}{(2 \pi)^{4}} k_{\mu} k_{\nu} e^{s k^{2}}=f(s) \eta_{\mu \nu}
$$

e contraindo $\mu$ e $\nu$, temos:

$$
\begin{aligned}
& \int \frac{d^{4} k}{(2 \pi)^{4}} k_{\mu} k^{\mu} e^{s k^{2}}=\int \frac{d^{4} k}{(2 \pi)^{4}} k^{2} e^{s k^{2}} \\
& =f(s) \eta_{\bullet \mu}^{\mu}=4 f(s) .
\end{aligned}
$$


Assim, obtemos uma forma de calcular $\mathrm{f}(\mathrm{s})$, pois $f(s)=\frac{1}{4} \int \frac{d^{4} k}{(2 \pi)^{4}} k^{2} e^{s k^{2}}$.

Cálculo de $\int \frac{d^{4} k}{(2 \pi)^{4}} k^{2} e^{s k^{2}}$

Calculando as integrais em $\theta$ e $\phi$ e aplicando a rotação de Wick, temos:

$$
\begin{aligned}
& -\frac{i}{8 \pi^{2}} \int k^{2} k^{3} e^{-s k^{2}} d k=-\frac{i}{16 \pi^{2}} \int k^{2} k^{2} e^{-s k^{2}} d k^{2} \\
& =-\frac{i}{16 \pi^{2}} \int d k^{2}\left(\frac{d^{2}}{d s^{2}}\left(e^{-s k^{2}}\right)\right)=-\frac{i}{16 \pi^{2}} \frac{d^{2}}{d s^{2}} \int e^{-s k^{2}} d k^{2} \\
& =\frac{-i}{16 \pi^{2}} \frac{d^{2}}{d s^{2}}\left(\frac{1}{s}\right)=-\frac{2 i}{16 \pi^{2} s^{3}}=-\frac{i}{8 \pi^{2} s^{3}} .
\end{aligned}
$$

Com isso, verificamos que

$$
f(s)=\frac{1}{4} \cdot\left(-\frac{i}{8 \pi^{2} s^{3}}\right)=-\frac{i}{32 \pi^{2} s^{3}}
$$

portanto

$$
\int \frac{d^{4} k}{(2 \pi)^{4}} k_{\mu} k_{\nu} e^{s k^{2}}=-\frac{i}{32 \pi^{2} s^{3}} \eta_{\mu \nu}
$$

Para a integral $\int k_{m u} k_{\nu} k_{\rho} k_{\sigma}$ o procedimento é o mesmo, exceto que desta vez o resultado terá um tensor de quarta ordem totalmente simétrico. Usando o anzatz:

$$
\int \frac{d^{4} k}{\left(2 \pi^{4}\right.} k_{\mu} k_{\nu} k_{\rho} k_{\sigma} e^{s k^{2}}=f(s)\left(\eta_{\mu \nu} \eta_{\rho \sigma}+\eta_{\mu \rho} \eta_{\nu \sigma}+\eta_{\mu \sigma} \eta_{\nu \rho}\right)
$$

e procedendo de modo semelhante ao caso anterior, obtemos:

$$
\int \frac{d^{4} k}{\left(2 \pi^{4}\right.} k_{m u} k_{\nu} k_{\rho} k_{\sigma} e^{s k^{2}}=\frac{i}{64 \pi^{2} s^{4}}\left(\eta_{\mu \nu} \eta_{\rho \sigma}+\eta_{\mu \rho} \eta_{\nu \sigma}+\eta_{\mu \sigma} \eta_{\nu \rho}\right)
$$




\section{Referências Bibliográficas}

[1] Kostelecky; V. A. and Samuel; S. Spontaneous breaking of lorentz symmetry in string theory. Physical Review D, 39:683-685, Junho 1988.

[2] Gomes; Marcelo O. C. Teoria Quântica dos Campos. EDUSP, São Paulo, 2002.

[3] Itzykson; Claude and Zuber; Jean-B. Quantum Field Theory. McGrawHill International Book Co., New York, 1980.

[4] Colladay; D. and Kostelecky; V. A. Cpt violation and the standard model. Physical Review D, 55:6760-6774, Janeiro 1997.

[5] Filho; Fernando T. da Silva. Correções Radiativas na Teoria de ChernSimons Calculadas em um Gauge Interpolante. Dissertação de mestrado. IFUSP, 2002.

[6] da Silva; Adilson J., Petrov; Albert, Nascimento; José R., Santos; Liner S., and Mariz; Tiago. Lorentz violation and the proper-time method. Physics Letters B, 661(4), Março 2008. 
[7] da Silva; Adilson J., Gomes; Marcelo, Nascimento; José R., Passos; E., and Petrov; Albert Yu. Induction of the four-dimensional lorentz-breaking non-abelian chern-simons action. Physics Review D, 76(047701), Agosto 2007.

[8] Jackiw; R. Deser; S. and Templeton; S. Topologically massive gauge theories. Annals of Physics, 140:372-411, Janeiro 1982.

[9] Carroll; S. M. et al. Limits on a lorentz and parity-violating modification of electrodynamics. Physics Letters B, 41(4), Fevereiro 1990.

[10] Adam; Madge G., Synge; J. L., Mandelstam; S., Essen;L., Sciama; D. W., Whitrow; G. J., and Weinberg; Steven. The observational tests of gravitation theory. Proceedings of the Royal Society of London. Series A, Mathematical and Physical Sciences, 270:297-305, Maio 1962.

[11] Kane; Gordon. Scientific American (Brasil), 39:100, 2005.

[12] Schwinger; Julian. On gauge invariance and vacuum polarization. Physical Review, 82:664-679, Dezembro 1951.

[13] Wiese; W. L. and Kelleher; D. E. On the cause of the redshifts in whitedwarf spectra. Astrophysical Journal, 166:L59-L63, Abril 1971.

[14] Peskin; Michael and Schroeder; David V. An Introduction to Quantum Field Theory. Westview press., EUA, 1995. 
[15] Jackiw; Roman. When radiative corrections are finite, but undetermined. International Journal of Modern Physics, B14:2011-2022, Agosto 2000.

[16] Ojima; Shuichi. Derivation of gauge and gravitational induced chernsimons terms in three dimensions. Progress of Theoretical Physics, 81(2), Fevereiro 1989.

[17] Weinberg; Steven. Gravitation and Cosmology: Principles and Applications of the General Theory of Relativity. John Wiley and Sons, Inc., Massachusetts, 1972.

[18] Mariz; Tiago, Nascimento; José R., Passos; E., and Ribeiro; R. F. Chernsimons-like action induced radiatively in general relativity. Physical Review D, 70(024014), Julho 2004.

[19] Dunne; Gerald V. Aspects of chern-simons theory. arXiv:hepth/9902115v1, 1999.

[20] Greiner; Walter and Reinhardt; Joachim. Field Quantization. SpringerVerlag, Frankfurt, 1996. 